\title{
Contribution of FE and FFT-based methods to the determination of the effective elastic and conduction properties of composite media with flat inclusions and infinite contrast
}

\author{
Joffrey Bluthée, ${ }^{\mathrm{a}, *}$, Benoît Bary ${ }^{\mathrm{a}}$, Eric Lemarchand ${ }^{\mathrm{b}}$ \\ ${ }^{a}$ Université Paris-Saclay, CEA, Service d'Étude du Comportement des Radionucléides, 91191, Gif-sur-Yvette, France \\ ${ }^{b}$ Laboratoire Navier - UMR 8205, CNRS, École des Ponts ParisTech, France
}

\begin{abstract}
Homogenization theory is increasingly applied to coupled phenomena, i.e. when different physical processes have to be modeled in order to correctly describe a system. The reason is that this methodology provides the means to propose a consistent morphological description of the system irrespective of the different phenomena involved, which is deemed to be physically sound. Here, we perform numerical simulations of both mechanical and transport processes in a linear context, so as to identify the best homogenization scheme out of five classical ones. The goal is to eventually apply the result to cracked rocks, which present complex structures, but this study is restricted to the case of parallel disks in an otherwise isotropic matrix. Since cracks in rocks present a vanishing stiffness but an infinite conductivity with respect to the rock, both types of contrast will be considered, and the reverse cases as well (infinite stiffness and vanishing conductivity) for the sake of completeness.
\end{abstract}

After detailing the motivations behind this work, the theoretical background necessary to derive all the analytical estimates is laid down. The derivations given are somewhat improved over previously published ones, and the framework is extended to deal with vanishing as well as infinite contrast. The methodology is explained for the 3D numerical simulations, and the results are presented and discussed. Two different numerical strategies have been used: an FEM software and an FFT-based code. This allows to lessen potential biases of a particular method, and increases the credibility of the results. All in all, the differential scheme is identified as the best fit, which confirms the results of previous studies, but this time in several different cases.

Keywords: Finite Element Method, Fast Fourier Transforms, Heterogeneous media, Cracked 
media, Linear elasticity, Conduction

\section{Introduction}

In many applications related to cracked rocks and soils, such as oil extraction, $\mathrm{CO}_{2}$ storage and radioactive waste storage, there is a deep connection between the mechanical state of the system and its fluid transport properties. This coupling comes in several forms: increased hydraulic conductivity as a consequence of the mode I nucleation and propagation of cracks, further increase caused by high pressures in the crack network, decrease of said conductivity due to clogging or mechanical closure of the cracks, etc... For these applications, it is necessary to possess a mathematical framework that takes this coupling into account. The field of micromechanics provides a way to introduce the hydromechanical coupling in a natural way, since the transport properties may be calculated from the knowledge of the geometrical evolution of the cracks, which can be obtained from the strain concentration rule. This approach has been used in recent years by a number of authors, such as Dormieux and Kondo [14], Barthelemy [4], Lemarchand et al. [29] and Levasseur et al. [30] among others. Most of the previous efforts were focused on the increased hydraulic conductivity caused by the propagation and/or opening of cracks.

On the other hand, the phenomenon of conductivity reduction as a consequence of mechanical loading or swelling of the rock has also received a lot of attention in the past two decades. This so-called "self-sealing" of swelling claystones is of great importance in the context of radioactive waste storage, since it is expected to play a major role in the safety assessment of deep repositories. As a consequence, a number of experiments have been carried out to investigate the conditions of occurrence of this process (see Blümling et al. [5], Ghayaza et al. [21], Davy et al. [12], Zhang [38] and Zhang [39] for examples of experiments and Bock et al. [8] for an overview of the investigations up until 2010). However, few attempts at modeling this phenomenon have been made up to now. Bluthe et al. [6] proposed to tackle this question at the scale of a drift using a micromechanical approach in a completely linear context, i.e. the cracks are assumed to remain open. This work

\footnotetext{
${ }^{*}$ Corresponding author

Email address: joffrey.bluthe@cea.fr (Joffrey Bluthé)
} 
was based on the model of a cracked rock introduced in Deude et al. [13], where a Mori-Tanaka scheme was used for the homogenization procedure. At this stage, the micromechanical analysis was limited to the mechanical behavior of the rock, not its transport properties, so that only the geometrical evolution of said rocks was analyzed. Moreover, the basic motivation for sticking with the Mori-Tanaka scheme was its ability to account for some interaction between the cracks with a quite simple implementation strategy. In other words, no heed was paid to the applicability of this scheme in the present context.

The aim of this paper is precisely to remedy these shortcomings by extending the micromechanical analysis to the hydraulic conductivity of the rock, and by assessing the performance of five classical micromechanical estimates in comparison with numerical simulations. The idea is to confirm previous results obtained in the context of linear elasticity (Bluthe et al. [7]), and to see if the same scheme is selected in the case of linear conduction. This will allow for a consistent hydromechanical representation of the cracked rock at the scale of the structure in an upcoming contribution. Note that this approach however does not take into account the coupling with the fluid pressure through the Biot's coefficient. One of the key points in this context is that from a mechanical point of view, a crack can be treated as an elastic medium with vanishing stiffness, while from a fluid transport point of view, the conductivity of a crack is much larger than that of the surrounding rock. As a consequence, two different mathematical treatments are required, although they are analogous in every respect. Numerically speaking, the effective conductivity of cracked samples was more delicate to assess with one of the numerical methods (FFT, see below), because the computations did not converge easily. In order to show that this was merely due to the fact that the contrasts are reversed (vanishing stiffness VS infinite conductivity), and not because of an inherent difference between linear elasticity and conduction, the case of flat rigid inclusions and that of flat insulating inclusions were also investigated. This allowed for a more general and thorough investigation of the effective behavior of composite media with flat inclusions and infinite contrast.

To do so, two different numerical methods were used, namely the Finite Element Method (FEM) 
with Cast $3 \mathrm{M}^{1}$, and the Fast Fourier Transform (FFT) approach with AMITEX_FFTP ${ }^{2}$, so as to check for possible biases of using only one particular numerical method. FEM tools are ubiquitous nowadays in research and engineering, which is reflected in the literature by the fact that most of the recent comparisons between analytical estimates and numerical simulations for cracked media were based on FEM. Among these contributions, one can cite Dahm and Becker [11] and Charpin and Ehrlacher [10] in 2D, and Grechka [22], Vasylevskyi et al. [35] and Bluthe et al. [7] in 3D (see Bluthe et al. [7] for more details on these papers). The previously mentioned contributions, as well as others based on different techniques, showed the good performance of the differential scheme in the case of linear elasticity. Some papers claimed a better fit of the dilute scheme, but as explained in Bluthe et al. [7], these authors used a stress-based estimate without consideration of the fact that the dilute scheme only applies for small values of the crack density parameter. As a consequence, these estimates of the out-of-plane elastic moduli were actually Mori-Tanaka estimates instead of dilute estimates. More recently, softwares with algorithms based on the Fast Fourier Transform (FFT), originally proposed by Moulinec and Suquet [31], have also been used to study the effective behavior of cracked solids. Willot et al. [37] have used such a software to study the effective behavior of a medium with parallel cracks in plain strain conditions; Willot et al. [36] have investigated the performance of the self-consistent scheme for the determination of the effective behavior of a randomly cracked polycrystal in 3D; finally, Gasnier et al. [20] have compared the precision of FEM and FFT methods in the case of a single crack in 2D, and have then assessed the performance of the self-consistent and dilute schemes to predict the effective stiffness of a solid containing either aligned cracks or randomly oriented cracks in 3D. In the latter contribution, the authors concluded that Fourier-based methods can indeed be used to model cracked solids, even in the case of randomly oriented cracks.

In the present paper, we thus propose to confirm and extend the aforementioned results. Both FEM and FFT have been used for comparison on almost identical geometries, the differences being simply due to the different discretization methods. The effective behavior of a solid with randomly located parallel flat cylindrical inclusions has been assessed numerically in 3D, for linear elasticity

\footnotetext{
${ }^{1} \mathrm{http}: / / \mathrm{www}-$ cast $3 \mathrm{~m} . c e a \cdot f r$

${ }^{2} \mathrm{http}: / /$ www.maisondelasimulation.fr/projects/amitex/html/index.html
} 
and linear conduction, the latter of which has not been dealt with previously. In both cases, the contrast between the properties of the inclusions and those of the matrix was taken to be either vanishing (i.e. voids/insulating inclusions) or infinite (i.e. rigid/superconducting inclusions). This represents an original contribution of the present work, since these properties are usually investigated separately and with only one contrast (either vanishing of infinite), although some papers have considered both contrasts, as in Kushch and Sangani [27] in the case of conductivity. The results were systematically compared with five classical analytical estimates in order to select the best fit. The findings are presented as follows: in section 2, the theoretical framework used for the analytical calculations is laid down, with a focus on the concentration rules for vanishing and infinite contrasts; in section 3, the two numerical procedures are described in turn, and in each case the results for both contrasts are compared with the analytical estimates; at the end of section 3 , the pros and cons of using the two numerical methods for cracked media are listed, and conclusions are then drawn for future works. Although the original goal of the study was to make a contribution in the numerical assessment of the performance of micromechanical estimates of the effective properties of singular composites, the required theoretical developments and the way they are presented here might also be of use to other researchers in the field of micromechanics, because they allow for a somewhat simpler and more unified treatment of different cases.

\section{Elements of homogenization of composites with flat inclusions}

This section is devoted to laying down a unified mathematical framework for the determination of the effective properties of composites with flat inclusions and for both infinite and vanishing contrasts, in the context of both linear elasticity and heat conduction. As mentioned in the introduction, an effort was made to provide a unified and comprehensive presentation of the main results, which can be found, for the most part, here and there in the literature. Although the results are not groundbreaking, the author reckoned that some insights can be gained from the following presentation, which is why some details are given in the case of linear elasticity. Indeed, it is more direct and more general than what can be found in the above references, and most importantly the specific components of the tensors which are affected by the inclusions naturally emerge. These developments were greatly inspired by Dormieux et al. [16] and Dormieux and Kondo [15], who 
provided a comprehensive take on how to apply homogenization theory to cracked solids.

Even though flat rigid inclusions and flat insulating inclusions are not necessarily relevant for the study of cracked rocks that the authors are pursuing, these cases were deemed interesting in so far as they provided additional confirmation of the good performance of the differential scheme and of the numerical methods used. Also, one could think of real world applications such as the computation of the thermal conduction of a cracked rock with dry cracks (air is a good thermal insulator), or that of the stiffness of a nanocomposite with aligned graphene plates, in analogy with the use of carbon nanotubes in material science. Additionally, partially saturated cracks could be represented by insulating inclusions for the water transport properties of a rock, so that this case is not at all devoid of physical meaning, even in the context of cracked rocks.

\subsection{Linear elasticity}

In homogenization theory, cracked media are often considered to be a limit case of a two-phase linear elastic composite whereby spheroidal inclusions with semi-axes $a$ and $c \ll a$ and stiffness tensor $\mathbb{C}^{I}$ are embedded in a matrix with stiffness tensor $\mathbb{C}^{m}$. A medium with open cracks is obtained by letting the stiffness of the inclusions vanish, and then by letting their aspect ratio $X=\frac{c}{a}$ vanish as well, see Dormieux and Kondo [15] for instance. Note that the order is important because if $\mathbb{C}^{I}$ is invertible when taking the limit $X \rightarrow 0$, then the inclusions have no effect on the effective stiffness of the composite. In other words, the stiffness tensor of the inclusions necessarily has to be singular for them to have an effect in the flat limit (see Barthelemy [2]). In practical applications, the stiffness of the inclusions will not be exactly zero for numerical purposes. Accordingly, a cracked medium will be obtained when $\left\|\mathbb{C}^{I}: \mathbb{S}^{m}\right\| \ll 1$ (where $\|\bullet\|$ is any norm on the space of fourth rank tensors), and the inclusions are said to be void. The reverse case, i.e. rigid inclusions, is characterized by $\left\|\mathbb{C}^{I}: \mathbb{S}^{m}\right\| \gg 1$, or equivalently $\left\|\mathbb{C}^{m}: \mathbb{S}^{I}\right\| \ll 1$. Note that the present developments are restricted to the case of parallel circular disks in an isotropic matrix, but further generalizations are possible (e.g. disks with different orientations in an anisotropic matrix). They would, however, require heavier numerical treatments. As a consequence, all the effective media obtained here are transversely isotropic.

The homogenization procedure is performed by prescribing specific boundary conditions to 
a Representative Elementary Volume (REV), assumed to contain all the necessary information about the composite body. The classical boundary conditions include: uniform strain boundary conditions, uniform stress boundary conditions and periodic boundary conditions. They all lead to a linear elastic effective medium and are asymptotically equivalent, i.e. they lead to the same homogenized stiffness $\mathbb{C}^{\text {hom }}$ when the size of the REV is much larger than the size of the heterogeneity. However, some choices are more convenient than others, as will be detailed in the sequel. In the following, the "microscopic" displacement, strain, stress, stiffness and compliance fields will be denoted $\boldsymbol{\xi}(\boldsymbol{z}), \boldsymbol{\varepsilon}(\boldsymbol{z}), \boldsymbol{\sigma}(\boldsymbol{z}), \mathbb{C}(\boldsymbol{z})$ and $\mathbb{S}(\boldsymbol{z})$ respectively, while the "macroscopic" strain, stress, stiffness and compliance tensors will be denoted $\boldsymbol{E}, \boldsymbol{\Sigma}, \mathbb{C}^{\text {hom }}$ and $\mathbb{S}^{\text {hom }}$ respectively. Here and in the following, $z$ is the position vector at the microscopic scale in the composite. Finally, the disk density parameter (DDP) $\epsilon=\mathcal{N} a^{3}$ is introduced in analogy with the crack density parameter (CDP) of Budiansky and O'Connell [9], where $\mathcal{N}$ is the number of inclusions per unit volume of composite, and all the estimates will be expressed as a function of this parameter. This quantity will be useful upon taking the limit $X \rightarrow 0$ of flat spheroids. In the case of voids, it does indeed have the usual interpretation of a CDP, but not in the case of rigid inclusions, which is why a more general denomination will be used.

\subsubsection{Flat voids}

Consider the case of uniform stress boundary conditions:

$$
\sigma(z) \cdot \boldsymbol{n}(z)=\boldsymbol{\Sigma} \cdot \boldsymbol{n}(z) ; \quad z \in \partial \Omega
$$

where $\partial \Omega$ is the boundary of the body $\Omega$. The boundary value problem is then linear in the loading parameter $\boldsymbol{\Sigma}$, so that a "strain concentration" tensor $\mathbb{A}^{\prime}(z)$ may be introduced such that:

$$
\varepsilon(z)=\mathbb{A}^{\prime}(z): \Sigma
$$

It will be shown that it is indeed convenient to introduce $\mathbb{A}^{\prime}(z)$ instead of the more widely used $\mathbb{A}(z)$ which relates the strain field to the macroscopic strain tensor. Defining the latter as $\boldsymbol{E}=\overline{\boldsymbol{\varepsilon}}$,

where $\bar{\bullet}=\frac{1}{|\Omega|} \int_{\Omega} \bullet \mathrm{d} V$ is the average operator over the whole REV, it follows that the effective compliance may be defined as:

$$
\mathbb{S}^{h o m}=\overline{\mathbb{A}^{\prime}}=f{\overline{\mathbb{A}^{\prime}}}^{I}+(1-f){\overline{\mathbb{A}^{\prime}}}^{m}
$$


where the volume fraction of inclusions $f$ has been introduced, as well as the volume average operators over each phase. Since the linear elastic constitutive relation reads $\sigma(z)=\mathbb{C}(z): \varepsilon(z)$, the uniform stress boundary conditions (1) yield:

$$
\Sigma=\bar{\sigma}=\overline{\mathbb{C}: \mathbb{A}^{\prime}}: \Sigma
$$

which holds for any symmetric tensor $\boldsymbol{\Sigma}$. Consequently, the following relation is obtained:

$$
f \mathbb{C}^{I}:{\overline{\mathbb{A}^{\prime}}}^{I}+(1-f) \mathbb{C}^{m}:{\overline{\mathbb{A}^{\prime}}}^{m}=\mathbb{I}
$$

where $\mathbb{I}$ is the fourth rank symmetric identity tensor. The tensor $\overline{\mathbb{A}}^{\prime}$ may then be eliminated from (3), which becomes:

$$
\mathbb{S}^{h o m}=\mathbb{S}^{m}+f\left(\mathbb{I}-\mathbb{S}^{m}: \mathbb{C}^{I}\right): \overline{\mathbb{A}}^{I}
$$

For spheroidal voids, $f$ is obtained from the DDP and the aspect ratio through the relation $f=\frac{4 \pi}{3} \epsilon X$. Also, one has in this particular case $\left\|\mathbb{S}^{m}: \mathbb{C}^{I}\right\| \ll 1$. The previous equation then becomes:

$$
\mathbb{S}^{h o m}=\mathbb{S}^{m}+\frac{4 \pi}{3} \epsilon X \overline{\mathbb{A}}^{I}
$$

This is an exact definition of the effective compliance tensor of a two-phase composite, which requires a priori an exact knowledge of the microstructure (through the concentration tensor). In order to circumvent this difficulty, estimates are proposed (see section 2.3).

In order to do this, it is necessary to obtain estimates of the concentration tensor $\overline{\mathbb{A}^{\prime}} I$. These estimates are classically built using the solution to the problem of a single inclusion embedded in an infinite matrix (Eshelby [18]). In the particular case of an ellipsoidal inclusion, the strain field in the inclusion is actually uniform and is related to the macroscopic strain tensor through the socalled Hill's tensor $\mathbb{P}^{I, m}$ (Hill [24]). This tensor is a function of the inclusion shape (superscript $I$ ) and the stiffness of the matrix (superscript $m$ ). Its interpretation is straightforward: in the problem of an infinite linear elastic homogeneous body with stiffness tensor $\mathbb{C}^{0}$ subjected to a polarization stress $\tau(z)=\sigma^{*} \mathbb{1}_{I}(z)$, where $\mathbb{1}_{I}$ is the indicator function of an ellipsoidal domain $I$, and vanishing displacements at infinity, the resulting strain in $I$ is uniform and given by:

$$
\boldsymbol{\varepsilon}^{I}=-\mathbb{P}^{I, 0}: \boldsymbol{\sigma}^{*}
$$


This result may then be used in order to solve the so-called auxiliary problem of an ellipsoidal inclusion $I$ with stiffness tensor $\mathbb{C}^{I}$ embedded in a reference material with stiffness $\mathbb{C}^{0}$, with uniform auxiliary stress boundary conditions $\Sigma^{0}$ (see figure 1). The reference material and the

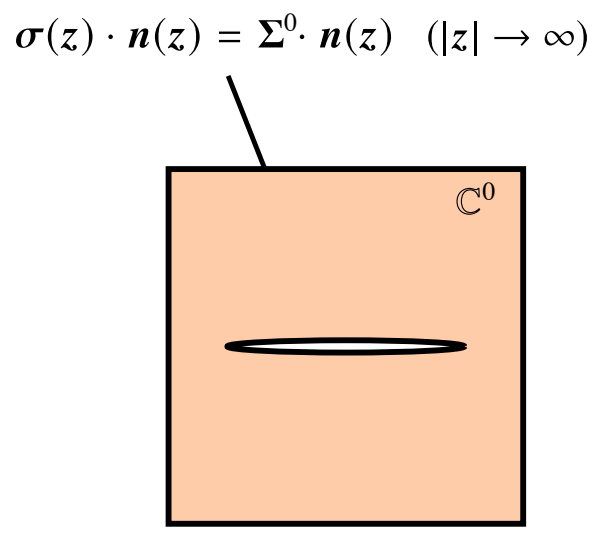

Figure 1: Auxiliary problem: uniform stress boundary conditions and reference material.

boundary condition $\Sigma^{0}$ can be chosen so as to take into account the interactions between the different inclusions. For this auxiliary problem, the strain field is again uniform in the inclusion and reads:

$$
\varepsilon^{I}=\left(\mathbb{C}^{0}+\mathbb{C}^{0}: \mathbb{P}^{I, 0}:\left(\mathbb{C}^{I}-\mathbb{C}^{0}\right)\right)^{-1}: \Sigma^{0}
$$

In the case of a void, this reduces to:

$$
\varepsilon^{I}=\left(\mathbb{Q}^{I, 0}\right)^{-1}: \Sigma^{0} ; \quad \mathbb{Q}^{I, 0}=\mathbb{C}^{0}-\mathbb{C}^{0}: \mathbb{P}^{I, 0}: \mathbb{C}^{0}
$$

In the sequel, the tensor $\mathbb{Q}^{I, 0}$ will be referred to as the second Hill's tensor (Hill [24]). Approximate solutions to the actual problem are then derived with appropriate values for $\mathbb{C}^{0}$ and $\Sigma^{0}$ (see section 2.3). As will be shown in section 2.3 , each of these choices leads to the introduction of a so-called interaction tensor (Dormieux and Kondo [15]) $\mathbb{M}$ such that $\boldsymbol{\Sigma}^{0}=\mathbb{M}: \boldsymbol{\Sigma}$, which depends on the scheme, so that:

$$
\lim _{X \rightarrow 0} X \overline{\mathbb{A}}^{I} \simeq \lim _{X \rightarrow 0} X\left(\mathbb{Q}^{I, 0}\right)^{-1}: \mathbb{M}
$$

Regardless of the scheme used in practice, it should be noted that $\boldsymbol{\varepsilon}^{I}$ becomes singular as $X \rightarrow 0$. This can be seen from the fact that the $O(1)$ terms in the expansion of $\mathbb{Q}^{I, 0}$ in powers of $X$ is singular. Indeed, its kernel can be shown to be $\mathcal{V}_{\text {oop }}^{2}=\operatorname{span}(\boldsymbol{n} \otimes \boldsymbol{n}, \boldsymbol{n} \stackrel{s}{\otimes} \boldsymbol{l}, \boldsymbol{n} \stackrel{s}{\otimes} \boldsymbol{m}$ ) (see Appendix 
A), where $\boldsymbol{n}$ is the unit normal to the flat void, $\boldsymbol{l}$ and $\boldsymbol{m}$ are two unit vectors such that $(\boldsymbol{n}, \boldsymbol{l}, \boldsymbol{m})$ is an orthonormal basis of the euclidean space, and $\stackrel{s}{\otimes}$ is the symmetric tensor product. Thus, $\mathcal{V}_{\text {oop }}^{2}$ is the linear subspace of out-of-plane symmetric second rank tensors with respect to the plane of the disks. However, one has the classical result that $X \boldsymbol{\varepsilon}^{I}$ has a finite limit as $X \rightarrow 0$ (see for instance Barthelemy [3]). Said limit is obtained from:

$$
\mathbb{U}^{d, 0}=\lim _{X \rightarrow 0} X\left(\mathbb{Q}^{I, 0}\right)^{-1}
$$

where the superscript $d$ stands for disk, since the limit $X \rightarrow 0$ has been taken. This tensor is computed from the $O(X)$ term in the expansion of $\mathbb{Q}^{I, m}$, as shown by the theorem proved in Barthelemy [3] (see appendix of that reference). The important result of the theorem is that $\mathbb{U}^{d, 0}$ is also singular, and its kernel is $\mathcal{V}_{i p}^{2}=\operatorname{span}(\boldsymbol{l} \otimes \boldsymbol{l}, \boldsymbol{m} \otimes \boldsymbol{m}, \boldsymbol{l} \stackrel{s}{\otimes} \boldsymbol{m})$, i.e. the linear subset of in-plane symmetric second rank tensors. Combining this result with (7), it appears that only the out-of-plane components of the effective compliance tensor will be affected by the presence of the flat voids, provided that the following two conditions are met by the interaction tensor:

- It has a finite limit as $X \rightarrow 0$

- It does not couple the in-plane and out-of-plane components of the stress tensor

These conditions will always be fulfilled in the cases under consideration here. On the other hand, the in-plane components of the effective stiffness tensor may still be affected because when inverting the compliance tensor, the $S_{3333}^{\text {hom }}$ component is necessarily mixed up with the in-plane components. The tensor $\mathbb{U}^{d, 0}$ is a function of $\mathbb{C}^{0}$ and the unit normal to the plane of the disk $\boldsymbol{n}$, and its Mandel representation is recalled in Bluthe et al. [7] for a transversely isotropic reference material.

Note that in Bluthe et al. [7], uniform strain boundary conditions were used, yielding equivalent results, which shows that the important part is to start from the microscopic strain and not the microscopic stress, irrespective of the boundary conditions. However, using uniform stress boundary conditions does present the advantage of being slightly more direct, since the effective compliance is naturally obtained whereas the effective stiffness had to be calculated first and then 
the relation had to be inverted. This was the main reason for the unusual way in which the general homogenization procedure was presented here.

\subsubsection{Flat rigid inclusions}

Let us now consider uniform strain boundary conditions, i.e. of the type:

$$
\boldsymbol{\xi}(z)=\boldsymbol{E} \cdot z ; \quad z \in \partial \Omega
$$

Then the boundary value problem is linear with respect to the loading parameter $\boldsymbol{E}$, so that a "stress concentration" tensor $\mathbb{B}^{\prime}(z)$ may be introduced, which satisfies:

$$
\sigma(z)=\mathbb{B}^{\prime}(z): E
$$

Following the same logic as in section 2.1.1, the effective stiffness is defined by $\mathbb{C}^{h o m}=\overline{\mathbb{B}^{\prime}}$ and the following relation is eventually obtained:

$$
\mathbb{C}^{\text {hom }}=\mathbb{C}^{m}+f\left(\mathbb{I}-\mathbb{C}^{m}: \mathbb{S}^{I}\right):{\overline{\mathbb{B}^{\prime}}}^{I}
$$

For spheroidal rigid inclusions one has $\left\|\mathbb{C}^{m}: \mathbb{S}^{I}\right\| \ll 1$, so that (15) becomes:

$$
\mathbb{C}^{h o m}=\mathbb{C}^{m}+\frac{4 \pi}{3} \epsilon X{\overline{\mathbb{B}^{\prime}}}^{I}
$$

An estimate for $\overline{\mathbb{B}^{\prime}}$ is then obtained in analogy with section 2.1.1. The result of Eshelby [18] is used with a prescribed stress-free strain $\varepsilon^{*}$ in the ellipsoidal domain $I$, so that the stress in this region is uniform and given by:

$$
\sigma^{I}=-\mathbb{Q}^{I, 0}: \varepsilon^{*}
$$

The auxiliary problem whereby uniform auxiliary strain boundary conditions $\boldsymbol{E}^{0}$ are applied to an infinite body containing an inclusion with stiffness tensor $\mathbb{C}^{I}$ can thus be solved. The stress in the inclusion is uniform and reads:

$$
\sigma^{I}=\left(\mathbb{S}^{0}+\mathbb{S}^{0}: \mathbb{Q}^{I, 0}:\left(\mathbb{S}^{I}-\mathbb{S}^{0}\right)\right)^{-1}: \boldsymbol{E}^{0}
$$

In the case of a rigid inclusion, this reduces to:

$$
\boldsymbol{\sigma}^{I}=\left(\mathbb{P}^{I, 0}\right)^{-1}: \boldsymbol{E}^{0}
$$


Approximate solutions to the actual problem are then obtained for specific choices of $\mathbb{C}^{0}$ and $\boldsymbol{E}^{0}$. Introducing again an interaction tensor $\mathbb{N}$, one has:

$$
\lim _{X \rightarrow 0} X{\overline{\mathbb{B}^{\prime}}}^{I} \simeq \lim _{X \rightarrow 0} X\left(\mathbb{P}^{I, 0}\right)^{-1}: \mathbb{N}
$$

The $O(1)$ term in the expansion of $\mathbb{P}^{I, 0}$ in powers of $X$ is also singular, but its kernel is $\mathcal{V}_{i p}^{2}$, so that $\sigma^{I}$ becomes singular as $X \rightarrow 0$. However, $X \sigma^{I}$ has a finite limit as $X \rightarrow 0$, which is obtained from:

$$
\mathbb{V}^{d, 0}=\lim _{X \rightarrow 0} X\left(\mathbb{P}^{I, 0}\right)^{-1}
$$

Similarly, $\mathbb{V}^{d, 0}$ is singular and its kernel is $\mathcal{V}_{\text {oop }}^{2}$, so that under the same assumptions concerning the interaction tensor as in section 2.1.1, it is seen from (16) that only the in-plane components of the stiffness tensor are affected by the presence of the rigid disks. Again, this does not hold for the compliance tensor. As previously stressed, this result is more readily obtained with the present original presentation than with the standard concentration tensors. The Mandel representation of $\mathbb{V}^{d, 0}$ is given in Appendix $\mathrm{B}$.

As could have been expected, the roles of stress/strain, stiffness/compliance, first/second Hill's tensor and in-plane/out-of-plane components are perfectly interchanged between flat voids and flat rigid inclusions. The symmetry between these problems is another reason for preferring the above presentation. A comment similar to the one made in section 2.1.1 can be also made here: the type of boundary conditions used is not really important, but starting from the microscopic stress and not the microscopic strain is. However, using uniform strain boundary conditions is slightly more direct for rigid inclusions.

\subsection{Linear conduction}

The case of linear conduction is treated by analogy with linear elasticity. Table 1 summarizes the connection between the quantities involved in the two cases. The notations and terminology of thermal conduction have been chosen, but note that all linear conduction laws (thermal, electric, hydraulic, diffusive) are mathematically identical. This choice merely comes from the fact that conduction is implemented in the context of thermal conduction in the softwares used. The constitutive relation of linear conduction $\operatorname{reads} \boldsymbol{q}=-\boldsymbol{K} \cdot \operatorname{grad} T$, so that the main difference 
between linear conduction and linear elasticity lies in the ranks of the tensors involved, since they are lower for linear conduction.

\begin{tabular}{c|c} 
Table 1: Correspondence between linear elasticity and linear conduction. \\
Linear elasticity & Linear conduction \\
\hline $\boldsymbol{\xi}$ (displacement vector) & $T$ (temperature) \\
$\boldsymbol{\varepsilon}$ (strain tensor) & $\boldsymbol{g r a d} T$ (gradient of temperature) \\
$\boldsymbol{\sigma}$ (stress tensor) & $\boldsymbol{q}$ (heat flux) \\
$\mathbb{C}$ (stiffness tensor) & $\boldsymbol{K}$ (conductivity tensor) \\
$\mathbb{S}$ (compliance tensor) & $\boldsymbol{R}$ (resistivity tensor)
\end{tabular}

The case of flat insulating inclusions is similar to that of flat voids in elasticity since one has $\left\|\boldsymbol{K}^{I} \cdot \boldsymbol{R}^{m}\right\| \ll 1$, so that uniform heat flux boundary conditions are applied at the boundary of the $\mathrm{REV}$, and a second rank concentration tensor $\boldsymbol{A}^{\prime}(\boldsymbol{z})$ for the gradient of temperature is introduced. After taking the limit $\left\|\boldsymbol{K}^{I} \cdot \boldsymbol{R}^{m}\right\| \rightarrow 0$, and then the limit $X \rightarrow 0$, one obtains:

$$
\boldsymbol{R}^{h o m}=\boldsymbol{R}^{m}+\frac{4 \pi}{3} \epsilon X{\overline{\boldsymbol{A}^{\prime}}}^{I}
$$

Estimates of the tensor ${\overline{\boldsymbol{A}^{\prime}}}^{I}$ may then be obtained using an auxiliary problem and the second Hill's tensor $\boldsymbol{Q}^{I, 0}$. Here, the second Hill's tensor is $\operatorname{singular}$ with $\operatorname{kernel} \mathcal{V}_{o o p}^{1}=\operatorname{span}(\boldsymbol{n})$, so that upon taking the limit $X \rightarrow 0$, one obtains a singular tensor $\boldsymbol{U}^{d, 0}=\lim _{X \rightarrow 0} X\left(\boldsymbol{Q}^{I, 0}\right)^{-1}$ with kernel $\mathcal{V}_{i p}^{1}=\operatorname{span}(\boldsymbol{l}, \boldsymbol{m})$, whose only non-zero component is given in Appendix C. Thus, the flat insulating inclusions only affect the out-of-plane resistivity.

Conversely, the case of flat superconducting inclusions is treated in analogy with that of flat rigid inclusions, since one has $\left\|\boldsymbol{K}^{m} \cdot \boldsymbol{R}^{I}\right\| \ll 1$. Uniform temperature gradient boundary conditions are applied at the boundary, and a second rank concentration tensor $\boldsymbol{B}^{\prime}(\boldsymbol{z})$ for the heat flux is introduced. One then takes the limit $\left\|\boldsymbol{K}^{m} \cdot \boldsymbol{R}^{I}\right\| \rightarrow 0$, and then $X \rightarrow 0$, which leads to:

$$
\boldsymbol{K}^{h o m}=\boldsymbol{K}^{m}+\frac{4 \pi}{3} \epsilon X{\overline{\boldsymbol{B}^{\prime}}}^{I}
$$


Here, estimates of the tensor ${\overline{\boldsymbol{B}^{\prime}}}^{I}$ may be obtained using an auxiliary problem and the traditional Hill's tensor $\boldsymbol{P}^{I, 0}$. This tensor is singular with kernel $\mathcal{V}_{i p}^{1}$, so that upon taking the limit $X \rightarrow 0$, one obtains a singular tensor $\boldsymbol{V}^{d, 0}=\lim _{X \rightarrow 0} X\left(\boldsymbol{P}^{I, 0}\right)^{-1}$ with kernel $\mathcal{V}_{\text {oop }}^{1}$, whose components are given in Appendix C. Thus, the flat superconducting inclusions only affect the in-plane conductivity.

\subsection{Homogenization schemes}

Five classical homogenization schemes have been chosen in the present work for comparison with numerical simulations. They are briefly presented hereafter in the case of rigid flat inclusions since they were already presented in Bluthe et al. [7] in the case of soft flat inclusions. The results obtained in the latter case, as well as the analogous results obtained for linear conduction, are given in Appendix D. The reader is reminded that we are only interested in parallel disks in the present contribution.

It has been stated here that rigid inclusions only affect the in-plane components of the stiffness tensor. To be more precise, the effect of the rigid inclusions may be reduced to the computation of two in-plane elastic moduli. Here, the in-plane shear modulus and P-wave modulus, defined in Appendix $\mathrm{B}$, have been chosen because the components of $\mathbb{V}^{d, 0}$ are most easily expressed using these moduli when $\mathbb{C}^{0}$ is no longer isotropic, as is shown in the same appendix.

\subsubsection{Dilute scheme}

The dilute approximation is obtained by setting $\boldsymbol{E}^{0}=\boldsymbol{E}$ and $\mathbb{C}^{0}=\mathbb{C}^{m}$ in (19). Then the interaction tensor is equal to the identity tensor $\mathbb{N}=\mathbb{I}$, which shows that no interaction between the inclusions is taken into account. The effective stiffness then reads:

$$
\mathbb{C}^{h o m} \simeq \mathbb{C}^{d i l}=\mathbb{C}^{m}+\frac{4 \pi}{3} \epsilon \mathbb{V}^{d, m}
$$

From Appendix B, the following effective moduli are obtained:

$$
\left\{\begin{array}{l}
\frac{M_{1}^{d i l}}{M_{m}}=1+\frac{16\left(11-14 v_{m}\right)\left(1-2 v_{m}\right)}{3\left(3-4 v_{m}\right)\left(7-8 v_{m}\right)} \epsilon \\
\frac{\mu_{12}^{d i l}}{\mu_{m}}=1+\frac{64\left(1-v_{m}\right)}{3\left(7-8 v_{m}\right)} \epsilon
\end{array}\right.
$$


where $M_{m}$ and $\mu_{m}$ are the P-wave and shear moduli of the matrix $\mathbb{C}^{m}$, and $v_{m}$ its Poisson's ratio. These are related by:

$$
\frac{M_{m}}{\mu_{m}}=\frac{2\left(1-v_{m}\right)}{1-2 v_{m}}
$$

Note that the dilute estimate of the effective compliance tensor is not $\left(\mathbb{C}^{d i l}\right)^{-1}$, as explained in Bluthe et al. [7], but its expansion to first order in $\epsilon$ :

$$
\mathbb{S}^{d i l}=\mathbb{S}^{m}-\frac{4 \pi}{3} \epsilon \mathbb{S}^{m}: \mathbb{V}^{d, m}: \mathbb{S}^{m}
$$

Indeed, the range of applicability of the dilute scheme is $\epsilon \ll 1$, so that one cannot simply invert (24). This result, together with (24), shows that the dilute estimates of the stiffness and compliance tensors are inverse to each other only to the second order in $\epsilon$, since one has:

$$
\mathbb{C}^{d i l}: \mathbb{S}^{d i l}=\mathbb{I}-\frac{16 \pi^{2}}{9} \epsilon^{2} \mathbb{V}^{d, m}: \mathbb{S}^{m}: \mathbb{V}^{d, m}: \mathbb{S}^{m}
$$

One should thus be careful when using this particular scheme to produce estimates. As an example, the reader is referred to the explanation regarding the effective Young's modulus and shear modulus in the aforementioned reference.

\subsubsection{Mori-Tanaka scheme}

Some interaction between the inclusions may be taken into account by setting $\boldsymbol{E}^{0}=\overline{\boldsymbol{\varepsilon}}^{m}$ and $\mathbb{C}^{0}=\mathbb{C}^{m}$. Then, the strain average rule $\overline{\boldsymbol{\varepsilon}}=\boldsymbol{E}$ yields $(1-f) \boldsymbol{E}^{0}=\boldsymbol{E}$, since $\boldsymbol{\varepsilon}=\mathbf{0}$ in the rigid inclusions. Thus, it turns out that the interaction tensor reduces to $\mathbb{N}=\frac{1}{1-f} \mathbb{I}$, and to $\mathbb{N}=\mathbb{I}$ when taking the limit $X \rightarrow 0$. As a consequence, the interaction between the inclusions vanishes in the limit $X \rightarrow 0$, and the dilute and Mori-Tanaka estimates of the effective stiffness are identical. This is analogous to a classical result in the case of cracks, whereby the dilute and Mori-Tanaka estimates of the effective compliance are identical. In the present case of flat rigid inclusions, the Mori-Tanaka estimate of the effective compliance is $\mathbb{S}^{M T}=\left(\mathbb{C}^{m}+\frac{4 \pi}{3} \epsilon \mathbb{V}^{d, m}\right)^{-1}$, so that it is different from the dilute estimate (27).

\subsubsection{Self-consistent scheme}

The self-consistent approximation consists in embedding the inclusions in the sought effective medium, that is in setting $\mathbb{C}^{0}=\mathbb{C}^{h o m} \simeq \mathbb{C}^{s c}$. This particular scheme is deemed able to account for 
a stronger interaction between the inclusions than the Mori-Tanaka scheme for a given value of $\epsilon$. Here, the self-consistent approximation is built by treating the composite as a dispersion of parallel rigid inclusions, as proposed by Hashin [23] in the case of cracked media. Additionally, $\boldsymbol{E}^{0}$ is set equal to $\boldsymbol{E}$. This yields the following implicit equation for the effective stiffness:

$$
\mathbb{C}^{s c}=\mathbb{C}^{m}+\frac{4 \pi}{3} \epsilon \mathbb{V}^{d, s c}
$$

Here, $\mathbb{V}^{d, s c}$ is a function of $\mathbb{C}^{s c}$, which is why (29) is actually implicit and must be solved numerically. Two implicit coupled equations are obtained and must be solved for $M_{1}^{s c}$ and $\mu_{12}^{s c}$, but they are not written down for the sake of simplicity.

\subsubsection{Differential scheme}

The differential scheme is another way to deal with strong interactions in composites with a high density of inclusions. It consists in building the composite in an iterative manner, by adding an infinitesimal amount $\mathrm{d} \mathcal{N}$ of inclusions per unit volume, leading to an increase in the DDP, here denoted by $e$, equal to $\mathrm{d} e=a^{3} \mathrm{~d} \mathcal{N}$. This leads to the following differential equation for the effective stiffness:

$$
\frac{\mathrm{d}}{\mathrm{d} e} \mathbb{C}^{d i f}=\frac{4 \pi}{3} \mathbb{V}^{d, d i f}
$$

Here, $\mathbb{V}^{d, d i f}$ is computed from $\mathbb{C}^{\text {dif }}$, so that it is a function of $e$, and this equation must be integrated from 0 to $\epsilon$ with the initial condition that $\mathbb{C}^{\operatorname{dif}}(0)=\mathbb{C}^{m}$. Thus, two coupled differential equations are obtained for $M_{1}^{d i f}$ and $\mu_{12}^{d i f}$, but they are not written down for the sake of simplicity.

\subsubsection{Ponte Castaneda and Willis bound}

An important class of estimates has been derived in Ponte Castaneda and Willis [33] from the Hashin-Shtrikman variational principles. These estimates, referred to as the PCW bounds, are able to account for specific types of inclusion distributions, those which possess ellipsoidal symmetry. They yield rigorous bounds on the effective stiffness in a certain range of inclusion densities. In the special case where the ellipsoid describing the distribution of inclusions is identical to the ellipsoidal shape of the inclusions themselves, the Mori-Tanaka estimate is recovered, so that this case will not be considered here. Another interesting case is that of a spherical distribution of 
inclusions. This is the one that will be referred to as PCW bound in the rest of the article. In the case of rigid inclusions, the resulting estimate is actually a lower bound for the exact effective stiffness. Formally, the estimate is still identical to the Mori-Tanaka estimate, but with a modified Hill's tensor, which reads:

$$
\mathbb{P}^{P C W}=\frac{1}{1-f}\left(\mathbb{P}^{I, m}-f \mathbb{P}^{\text {sphere }, m}\right)
$$

where $\mathbb{P}^{\text {sphere, } m}$ is the Hill's tensor for a sphere with the reference stiffness $\mathbb{C}^{m}$, i.e.:

$$
\mathbb{P}^{\text {sphere } m}=\frac{1}{3 M_{m}} \mathbb{J}+\frac{3 M_{m}+2 \mu_{m}}{15 \mu_{m} M_{m}} \mathbb{K}
$$

$\mathbb{J}$ and $\mathbb{K}$ being the spherical and deviatoric projectors. It is clear from (31) that the Hill's tensor of the sphere will impact the $O(X)$ term in the expansion of $\mathbb{P}^{P C W}$ in powers of $X$ because $f=\frac{4 \pi}{3} \epsilon X$. After some straightforward but tedious calculations, the following estimates are obtained:

$$
\left\{\begin{array}{l}
\frac{M_{1}^{P C W}}{M_{m}}=1+\frac{240 \epsilon\left(1-2 v_{m}\right)}{45\left(3-4 v_{m}\right)-64 \epsilon\left(3-5 v_{m}\right)}+\frac{480 \epsilon\left(1-2 v_{m}\right)}{45\left(7-8 v_{m}\right)-128 \epsilon\left(4-5 v_{m}\right)} \\
\frac{\mu_{12}^{P C W}}{\mu_{m}}=1+\frac{960 \epsilon\left(1-v_{m}\right)}{45\left(7-8 v_{m}\right)-128 \epsilon\left(4-5 v_{m}\right)}
\end{array}\right.
$$

These are rigorous lower bounds for $\epsilon \leq \frac{3}{4 \pi} \simeq 0.24$, and they exhibit a percolation threshold for $\epsilon=\frac{45\left(7-8 v_{m}\right)}{128\left(4-5 v_{m}\right)}$. When $v_{m}$ goes from 0 to 0.5 , the percolation threshold increases from $\epsilon \simeq 0.615$ to $\epsilon \simeq 0.703$, so that the status of bound is lost before this threshold is reached anyway.

\section{Numerical simulations}

The simulation step is closely related to the work presented in Bluthe et al. [7] (see this reference for details). Elementary Volumes (EVs) have been generated using the same procedure: the inclusion centroids are randomly distributed in a cubic box using the python tool Combs developed for the CAD platform Salome ${ }^{3}$, and the shape and size of the inclusions is prescribed. As in the aforementioned contribution, flat cylinders have been used instead of flat spheroids, as they make the geometry generation easier and this has a negligible impact on the results. Also, to further simplify the generation step, the inclusions were actually defined as prisms with 200-sided polygonal bases, since the circles are discretized during the meshing step anyway. Interpenetration

\footnotetext{
${ }^{3}$ https://www.salome-platform.org
} 
of the inclusions was allowed here, which was previously not the case, so that the positions of the centroids are independent. In order to improve on the results obtained in the above reference, an effort was made to increase the number of inclusions in each $\mathrm{EV}$, since it was deemed from statistical analysis that there weren't enough of them for the EVs to be representative. The same DDP range was explored ( 0.2 to 0.8 ), but the radius of the inclusions is about $a=0.159 L$ in the present study, where $L$ is the edge length of the $\mathrm{EV}$, while it ranged from $0.188 \mathrm{~L}$ to $0.237 \mathrm{~L}$ in Bluthe et al. [7], representing a 15-33\% decrease. This allowed for the number of inclusions to be increased from 30 to 50 for $\epsilon=0.2$, and from 60 to 200 for $\epsilon=0.8$ (and similarly for $\epsilon=0.4$ and $\epsilon=0.6$ ). For each value of the DDP, three different geometries were thus generated. Finally, periodic microstructures were prescribed, i.e. inclusions whose centroid is less than $a$ away from a side of the box reappear on the opposite side (see figure 2). That way, if the box is repeated periodically in space, all the inclusions are complete, and not cut.

The discretization procedures will be described in sections 3.1.1 and 3.2.1 since they depend on the specific numerical method. Periodic boundary conditions are necessarily applied to the EVs in the case of FFT, but they were also applied for the FEM simulations, since uniform boundary conditions were previously shown to yield unsatisfactory results with these geometries (see Bluthe et al. [7]). Note that periodic boundary conditions do not provide perfectly accurate results either, but at least they do yield intermediate results between uniform stress and uniform strain conditions. The size required for the elementary volume to actually representative is not easily determined beforehand, but there are some results in some specific cases, such as Drugan and Willis [17] in the case of an isotropic distribution of spheres in a matrix, both of which are made up of an isotropic material. For voids, the minimum requirement for the size of the elementary volume is a function of the Poisson's ratio of the matrix and of the inclusion volume fraction. In the present paper, the Poisson's ratio has been chosen as $v_{m}=0.2$, which is a usual value for rocks and concretes, and the inclusion volume fraction is given by $f=\frac{4 \pi}{3} \epsilon X$. As will be discussed below, $X$ will be lower than $6.3 \cdot 10^{-2}$ (and even lower than 0.01 in the case of FEM), so that the maximum crack volume fraction used here is 0.21. According to Drugan and Willis [17], this leads to the condition that $\frac{L}{2 a}$ should be greater that some number between 1.967 and 1.987 for an error limit of 5\%, if the inclusions were spheres. Of course the present contribution deals with disks instead of spheres, 
but it is worth noting that we have $\frac{L}{2 a} \simeq 3.14$, which is indeed greater than 1.987 .

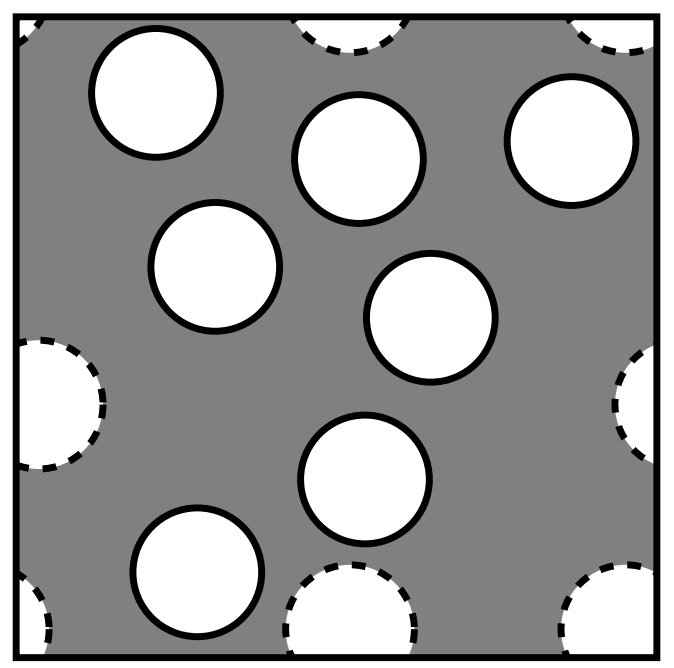

Figure 2: Example of a 2D periodic EV. Inclusions in solid lines are entirely inside the box; inclusions in dotted lines intersect with at least one of the boundaries and reappear on another side of the box.

\subsection{FEM}

\subsubsection{Method}

Automatic softwares plugged in Salome ${ }^{4}$ were used to generate periodic meshes from the geometries previously discussed. They are made up of tetrahedra for volumes and triangles for surfaces. Note that the mesh size was systematically reduced close to the inclusion edges as the fields are expected to present high gradients in these regions. An example is shown in figure 3.

\subsubsection{Flat voids and flat insulating inclusions}

In the case of soft flat inclusions, the out-of-plane components $S_{3333}$ and $S_{1313}$ of the compliance tensor are sought, where $\boldsymbol{e}_{3}$ has been chosen as the unit vector perpendicular to the plane of the inclusions. These components may be obtained for the different EVs by prescribing successively $\boldsymbol{\Sigma}=\boldsymbol{e}_{3} \otimes \boldsymbol{e}_{3}$ and measuring $E_{33}$, and $\boldsymbol{\Sigma}=\boldsymbol{e}_{1} \otimes \boldsymbol{e}_{3}+\boldsymbol{e}_{3} \otimes \boldsymbol{e}_{1}$ and measuring $E_{13}$. The results are presented, along with the micromechanical estimates, in terms of the out-of-plane Young's and shear moduli in figure 4, and the differential scheme is clearly the best fit. In figure 4, two different

\footnotetext{
${ }^{4}$ http://distene.com
} 

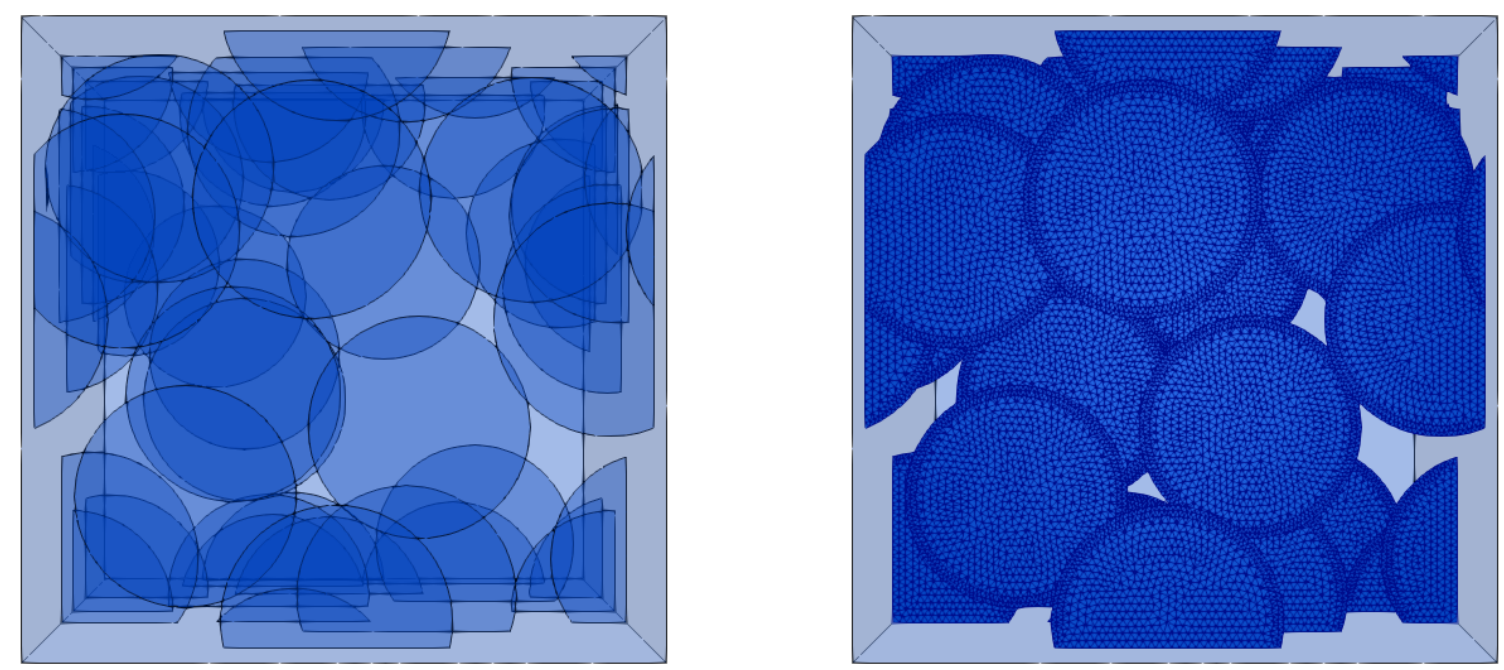

Figure 3: Top view of a generated geometry (left) and the associated mesh (right). Only the inclusions are shown for clarity. The mesh refinement close to the inclusion edges and the periodicity of the geometry can be seen.

values for the initial aspect ratio of the inclusions were used for $\epsilon=0.8$, namely $\frac{c}{a}=\frac{1}{1000}$ and $\frac{c}{a}=\frac{1}{100}$, so as to investigate the influence of this parameter. Since these geometries have the highest number of inclusions, the effect of the aspect ratio should be stronger than for the other values of the DDP. As can be seen, the influence on the results is very modest and still favors the differential scheme. These results were obtained with a finite, but very large, contrast of $5 \cdot 10^{7}$ between the Young's moduli of the two phases.

For insulating flat inclusions, only the out-of-plane component $R_{33}$ of the resistivity tensor is sought. It may be obtained by prescribing an average heat flux $\overline{\boldsymbol{q}}=\boldsymbol{e}_{3}$ and measuring $\overline{\frac{\partial T}{\partial z_{3}}}$. Once again, figure 5 shows that the differential scheme best fits the numerical simulations. In this case, all the geometries were tested with two levels of mesh refinements, so as to check for mesh convergence. Coarse meshes were used for the left-hand plot, with about 3 million elements for $\epsilon=0.2$ and almost 7 million elements for $\epsilon=0.8$, while fine meshes were used for the right-hand plot, with about 7 million elements for $\epsilon=0.2$ and over 17 million elements for $\epsilon=0.8$. The effect of such a refinement is not visible to the eye in the figure, which does indicate good mesh convergence. A contrast of $10^{10}$ between the conductivities of the two phases was used to obtain 


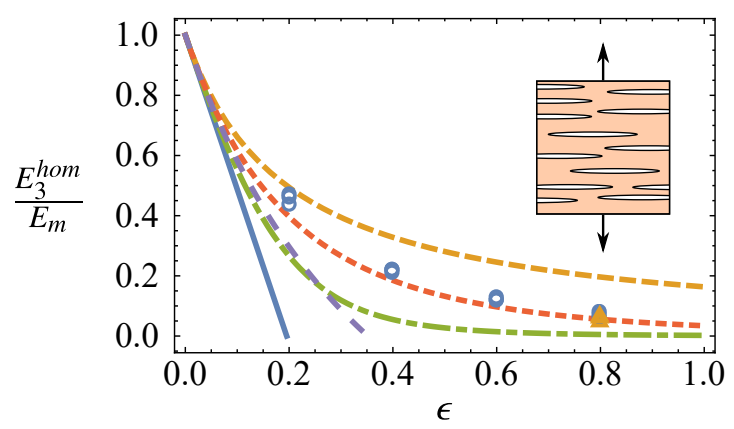

Dilute scheme

Differential scheme

$=-=-$ Mori-Tanaka scheme $\quad=-=$ PCW bound

- $=-$ Self-consistent scheme

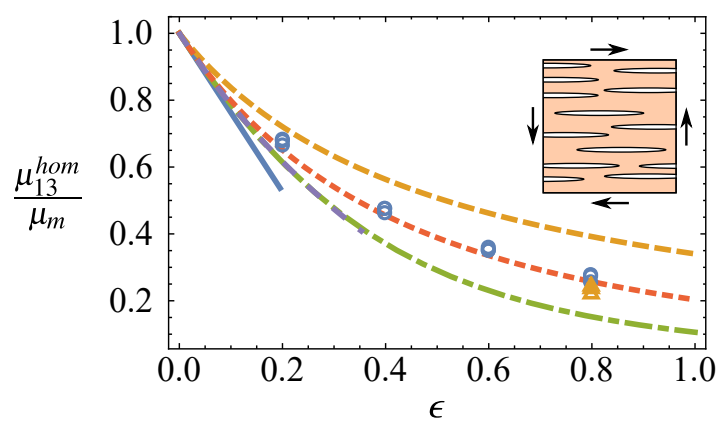

- FEM (aspect ratio 1/1000)

$\triangle$ FEM (aspect ratio $1 / 100)$

Figure 4: Out-of-plane Young's and shear moduli as a function of DDP: FEM vs analytical estimates for flat voids.

these results.

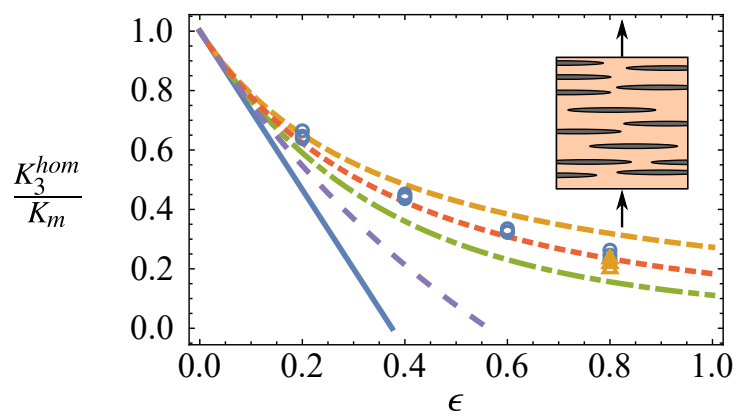

— Dilute scheme

---= Mori-Tanaka scheme $\quad=-=$ PCW bound

- $=$ Self-consistent scheme

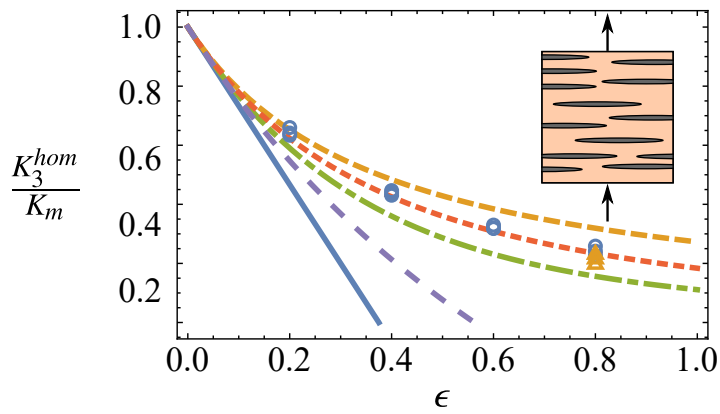

○ FEM (aspect ratio 1/1000)

$\triangle$ FEM (aspect ratio $1 / 100)$

Figure 5: Out-of-plane conductivity as a function of DDP: FEM vs analytical estimates for flat insulating inclusions. Left: coarse mesh; right: fine mesh.

\subsubsection{Flat rigid inclusions and flat superconducting inclusions}

A similar analysis is performed in the case of flat rigid inclusions and flat superconducting inclusions on the exact same geometries by inverting the contrast. More precisely, a contrast of $10^{8}$ was used for the elastic moduli, and $10^{10}$ for the conductivity. This time, the in-plane components of the stiffness and conductivity tensors respectively are sought. For flat rigid inclusions, the three in-plane components of the average stress tensor were successively prescribed in three different 
simulations, so that two values of the in-plane P-wave modulus could be obtained (one for each in-plane direction), as well as the in-plane shear modulus. These two values of the in-plane Pwave modulus allowed to show that consistent results are obtained when loading the two in-plane directions. More precisely, upon applying an average stress tensor of the type $\Sigma=\Sigma \boldsymbol{e}_{1} \otimes \boldsymbol{e}_{1}$, Hook's law yields:

$$
\left\{\begin{array}{l}
\Sigma=M_{1}^{h o m} E_{11}+\left(M_{1}^{h o m}-2 \mu_{12}^{h o m}\right) E_{22}+\lambda_{s} E_{33} \\
0=\left(M_{1}^{\text {hom }}-2 \mu_{12}^{\text {hom }}\right) E_{11}+M_{1}^{\text {hom }} E_{22}+\lambda_{s} E_{33}
\end{array}\right.
$$

where a transversely isotropic behavior of the EV has been assumed, use has been made of the fact that the 3333 component of the stiffness tensor is unaffected by the disks (see Appendix B), and $\lambda_{s}$ is the first Lamé coefficient of the matrix. This $2 \times 2$ system may then be inverted to yield:

$$
M_{1}^{\text {hom }}=\frac{\Sigma E_{11}-\lambda_{s}\left(E_{11}-E_{22}\right) E_{33}}{E_{11}^{2}-E_{22}^{2}}
$$

A similar derivation yields a value $M_{2}^{h o m}$ obtained upon application of an average stress tensor $\boldsymbol{\Sigma}=\Sigma \boldsymbol{e}_{2} \otimes \boldsymbol{e}_{2}$, and the result is the same as (35) with the indices 1 and 2 interchanged. In hindsight, it would have been more direct to prescribe the strain components instead of the stress components in this particular context, which would have spared us this step. For flat superconducting inclusions, an average temperature gradient of the form $\overline{\operatorname{grad} T}=\mathbf{1}$ was prescribed, so as to be able to compute all three components of the conductivity tensor.

The results are shown in figures 6 and 7. In each case, the figure on the left shows the results obtained in the two different directions with an aspect ratio of 1:1000, while the figure on the right shows the influence of the aspect ratio. In the case of linear elasticity, the latter influence was shown for the shear modulus, while the average in-plane conductivity was chosen in the case of linear conduction. It is clear from these two figures that the scatter in the results is greater than for flat voids and flat insulating inclusions, especially for $\epsilon=0.8$. However, the differential scheme can still be identified as the best fit. Note that the results obtained with an aspect ratio of 1:100 appear to be closer to the self-consistent scheme than to the differential scheme, but it can be seen that reducing the aspect ratio to 1:1000 brings the data points closer to the latter scheme. This point will be further expanded upon in the case of FFT simulations. Both left-hand plots show that the properties of the generated samples obtained in the directions $\boldsymbol{e}_{1}$ and $\boldsymbol{e}_{2}$ are indeed very close, 
which confirms the good transverse isotropy observed in Bluthe et al. [7] on different samples. Finally, note that the right-hand plot of figure 7 shows the average conductivity between the two directions, hence the " $\langle\bullet\rangle "$ symbols, so as to not clutter the plot with too many markers.
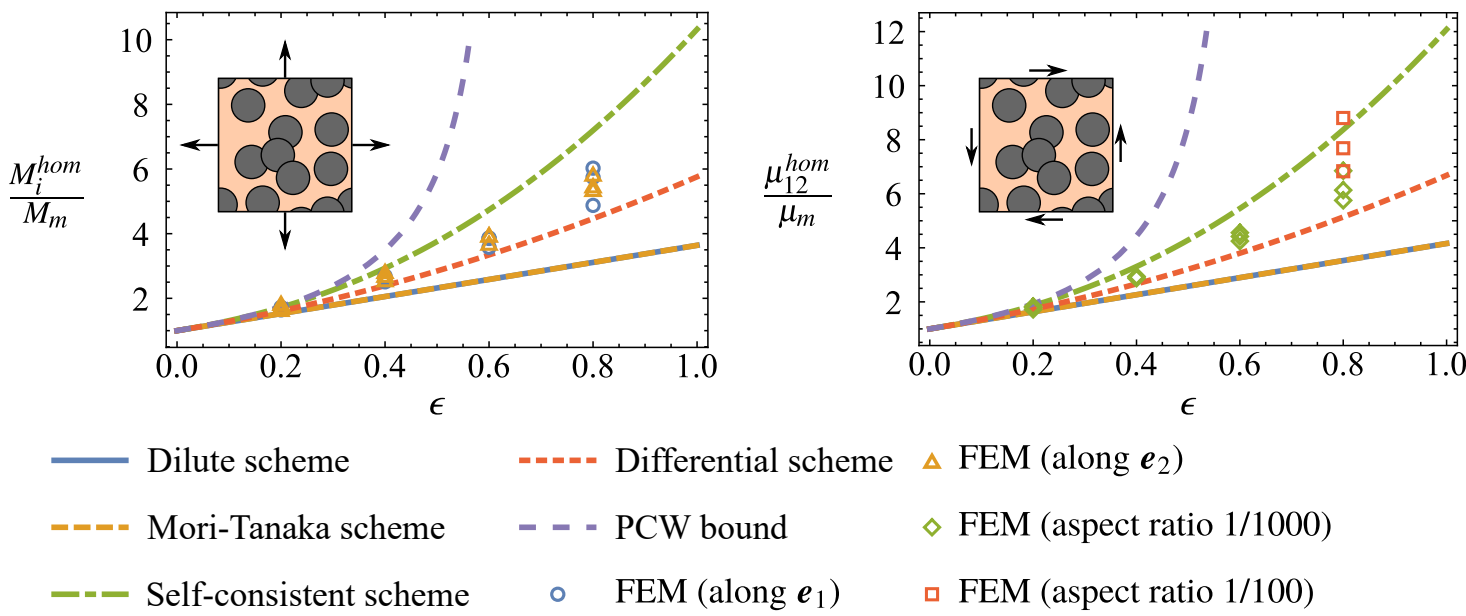

Figure 6: In-plane P-wave and shear moduli as a function of DDP: FEM vs analytical estimates for flat rigid inclusions.
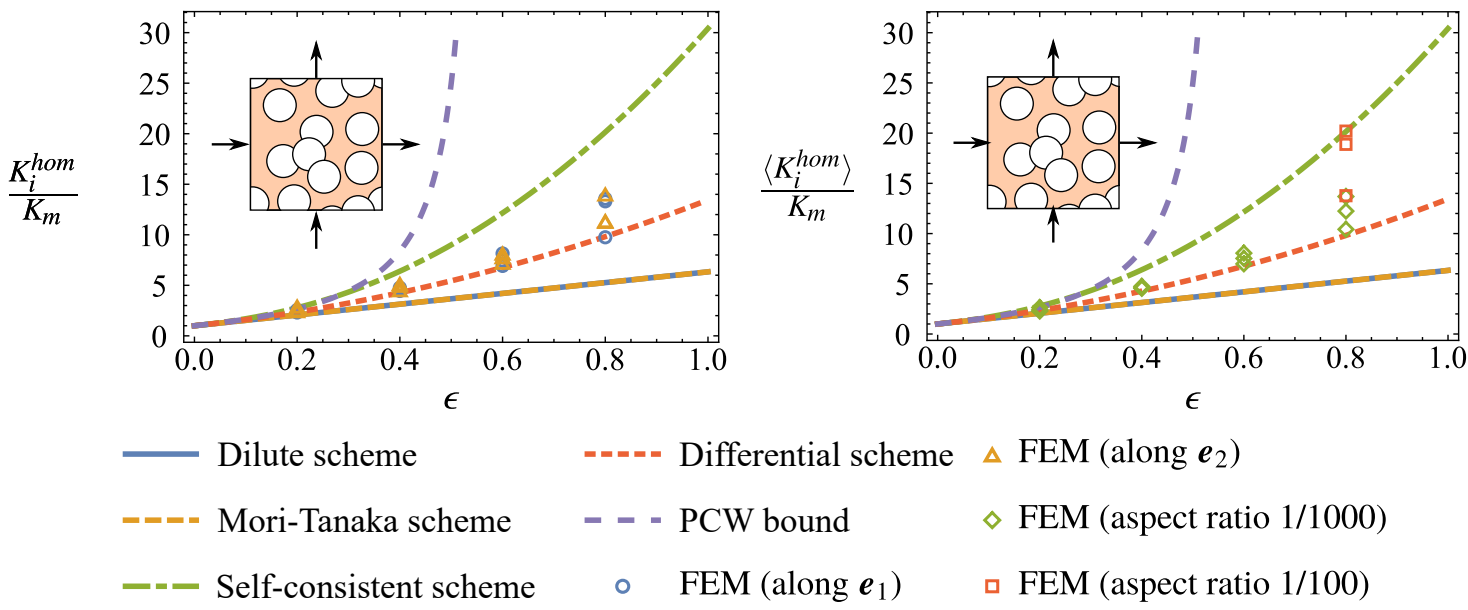

Figure 7: In-plane conductivities as a function of DDP: FEM vs analytical estimates for flat superconducting inclusions. Left: both in-plane directions; right: influence of the aspect ratio of the inclusions. 


\section{2. $F F T$}

\subsubsection{Method}

The geometries previously generated for FEM calculations were then discretized using voxels in order to use the FFT-based software AMITEX_FFTP ${ }^{5}$, in combination with the FFT library fftw version 3.3.8. Regular grids were used, with three different levels of refinement: $50^{3}, 200^{3}$ and $500^{3}$ voxels respectively. In each case, the inclusions were represented as one-voxel thick cylinders with an approximately circular base. These thicknesses were 6.3:100, 1.6:100 and 6.3:1000 for the three grid sizes respectively. Of course, the base of the cylinder is more and more circular as the number of voxels increases. Note that an inherent characteristic of this discretization procedure is that the positions of the centroids of the inclusions are only respected to within a voxel, so that the positions of the inclusions are less precise with a coarser grid. Additionally, thicker inclusions are more likely to interpenetrate, so that percolation phenomena are more frequent with a coarser grid. That being said, it will be shown that certain properties are obtained with great precision, even with a coarse grid.

\subsubsection{Flat voids and flat insulating inclusions}

As in section 2.1.1, the EVs were subjected to uniaxial tension in the direction perpendicular to the plane of the inclusions to determine the out-of-plane Young's and shear moduli. A contrast of about $10^{9}$ between the matrix and the inclusions was used. The results are presented in figure 8 . The three different grid refinement levels have been plotted together with the analytical results, and for the Young's modulus the markers are surprisingly close. This means that even a very coarse grid may be used to determine the out-of-plane Young's modulus of such a composite. On the other hand, there is a distinct separation between the refinement levels when looking at the shear modulus. Still, the difference between the $200^{3}$ and $500^{3}$ grids is low enough to reckon that further refining the grid is unnecessary. Finally, the differential scheme is here again identified as the best fit for the finer grids.

Similar observation can be made for the determination of the out-of-plane conductivity of the composites (see figure 9): the markers corresponding to the different refinement levels may be

\footnotetext{
${ }^{5}$ http://www.maisondelasimulation.fr/projects/amitex/html/index.html
} 

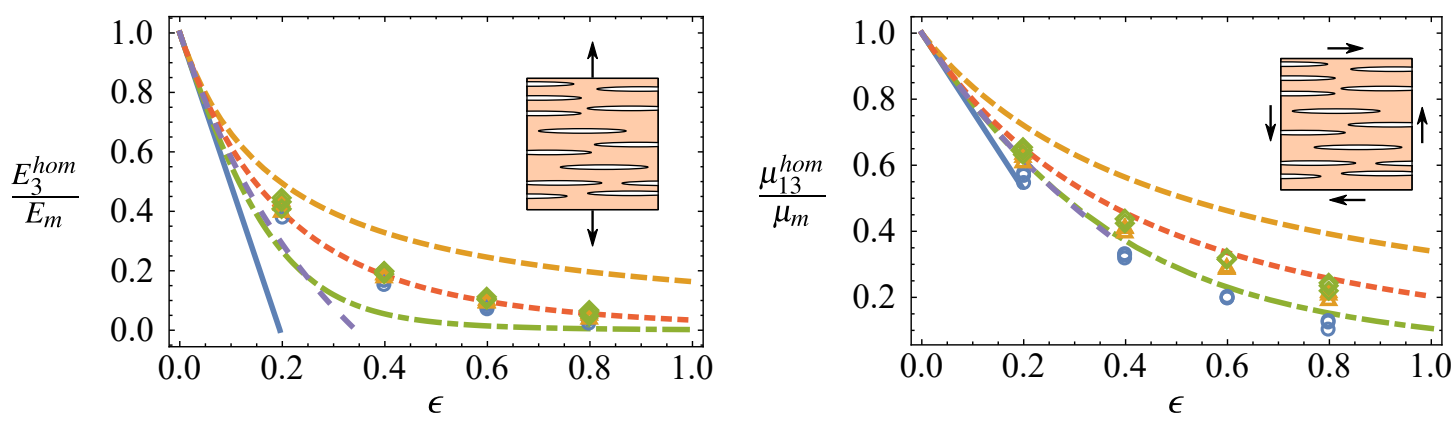

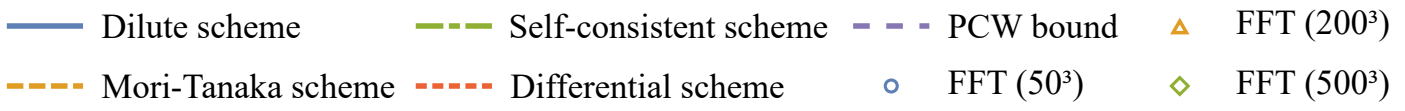

Figure 8: Out-of-plane Young's and shear moduli as a function of DDP: FFT vs analytical estimates for flat voids.

distinguished, but the difference between the results obtained with the $200^{3}$ and $500^{3}$ grids is small enough that these can be trusted. Thus, the differential scheme yields the best fit.

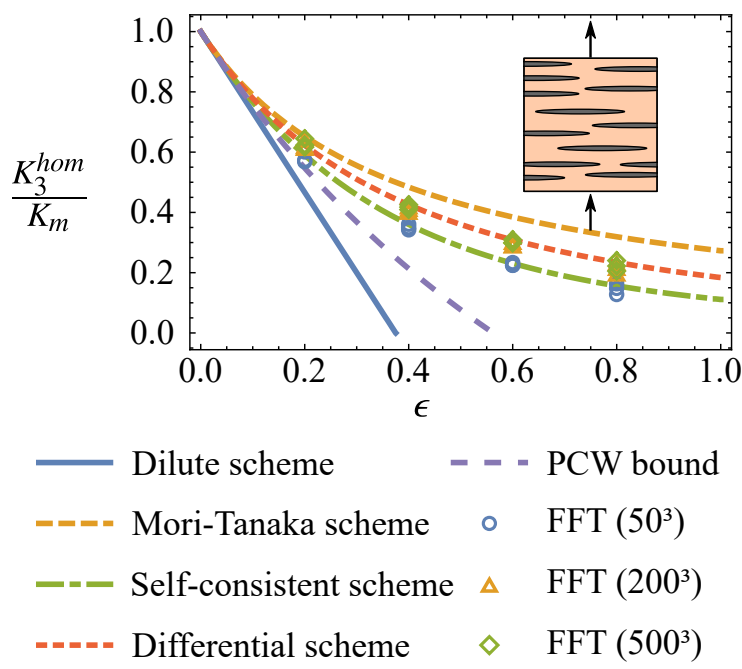

Figure 9: Out-of-plane conductivity as a function of DDP: FFT vs analytical estimates for flat insulating inclusions.

\subsubsection{Flat rigid inclusions and flat superconducting inclusions}

The case of flat rigid inclusions proved to be more difficult with FFT, since percolation phenomena were observed for reasons mentioned in 3.2.1. Using very large contrasts prevented the simulations from converging, so that a contrast of only $10^{5}$ eventually had to be used. Even then, only the finest grid yielded usable results for all the simulations, as can be seen in figure 10. Indeed, the results obtained with the $50^{3}$ grids were way above the curves for $\epsilon=0.8$ and are thus not 
represented. The same phenomenon appeared for one out of the three $200^{3}$ grids. This time, the loading was applied only in the $\boldsymbol{e}_{1}$ direction because the same geometries were used as before and the FEM simulations had already shown a satisfactory transverse isotropy. A large amount of scatter was observed between the simulations for $\epsilon \geq 0.6$, which does not allow for a clear decision to be made between the self-consistent and differential schemes. However, one can note that increasing the grid size tends to lower the results, so that one can speculate that further refining the grid would eliminate the self-consistent scheme from the potential candidates. All-in-all, these numerical results do not discard the differential scheme, which is still the best fit for $\epsilon \leq 0.6$.

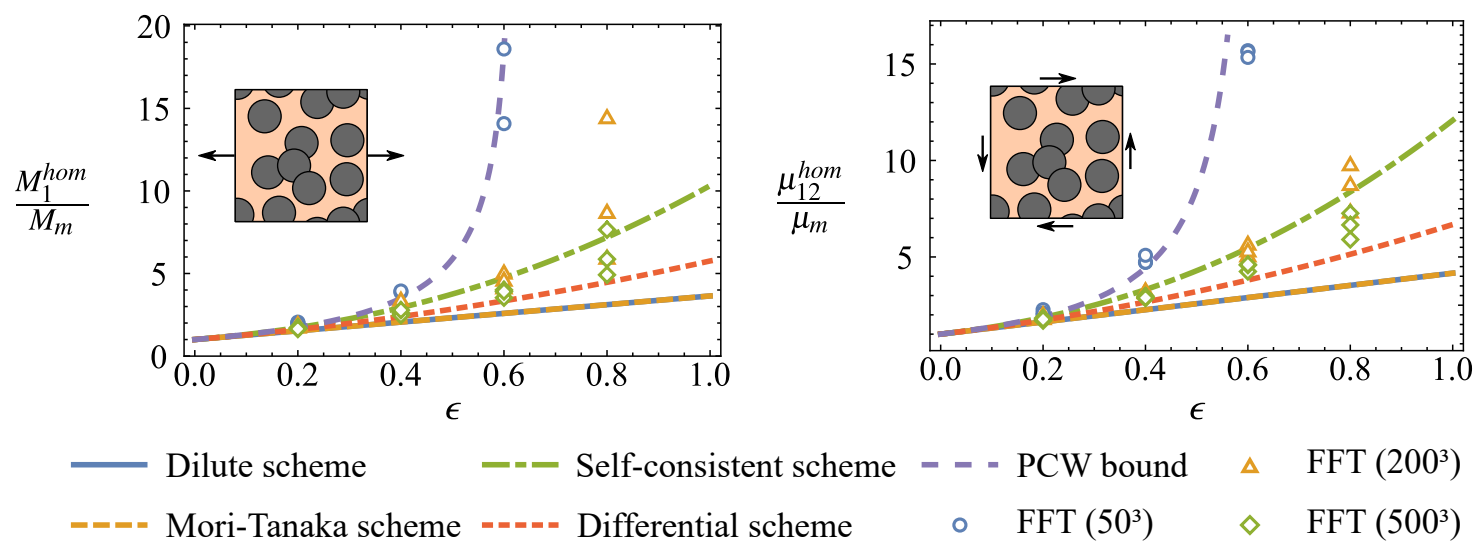

Figure 10: In-plane P-wave and shear moduli as a function of DDP: FFT vs analytical estimates for flat rigid inclusions.

Similar trends were observed in the context of superconducting inclusions. The in-plane conductivity was susceptible to percolation, but here it was possible to get the computations to converge even with a contrast of $10^{10}$. As can be seen from figure 11, very few results are presented for the $50^{3}$ grid, and those obtained with the $500^{3}$ grid lie between the self-consistent and differential schemes. Again, the latter scheme does appear to best fit the numerical data for $\epsilon \leq 0.6$, and the general trend pushes toward speculating that using even finer grids would favor this scheme for $\epsilon=0.8$ as well. Keep in mind that the aspect ratio of the cracks is dictated by the grid size, and the aspect ratio of 1:1000 used in the FEM simulations could not be reached, even with the $500^{3}$ grid.

\subsection{Comparison of the two numerical methods}

Both methods yielded similar results in terms of identifying one scheme that performs better than the others, but they do present some differences that should be pointed out for future 


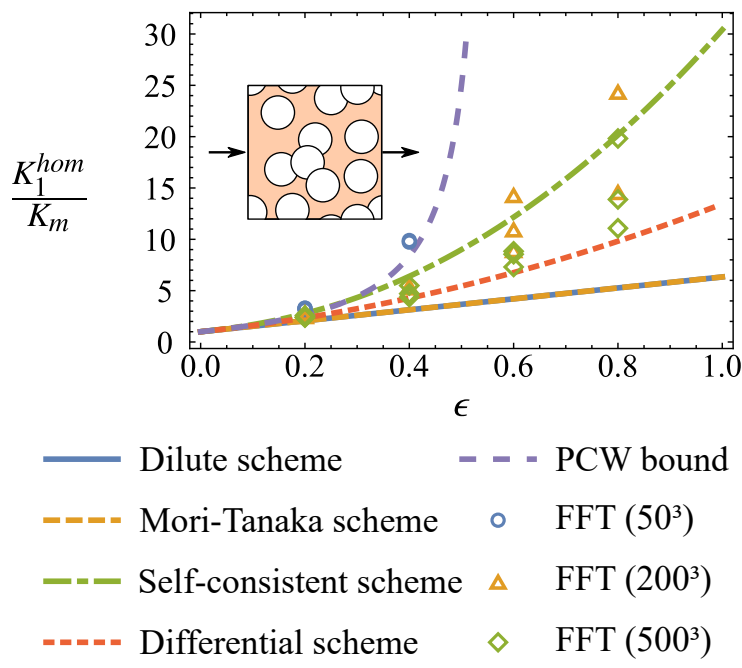

Figure 11: In-plane conductivity as a function of DDP: FFT vs analytical estimates for flat superconducting inclusions.

investigations of the sort. Let us summarize the pros and cons of using one versus the other.

FEM is ubiquitous nowadays in engineering, so it is no surprise that it has been used extensively for the determination of the effective properties of composites. With this method, it is possible to choose between uniform strain, uniform stress and periodic boundary conditions, which is a great advantage. Although periodic boundary conditions are often preferred because they yield more precise estimates for locally periodic composites (see Hollister and Kikuchi [25]), the possibility of prescribing uniform boundary conditions allows to assess if the EVs are representative or not. In terms of computation times, the mechanical simulations described here ran for about one and seven hours on a standard Linux machine with 48 cores and 48 GB for $\epsilon=0.2$ and $\epsilon=0.8$ respectively, while the longest heat conduction simulations ran for about an hour $(\epsilon=0.8)$. This difference is to be expected since there are fewer degrees of freedom for heat conduction than for linear elasticity. One important point is that the computation time was about the same whether one considered voids or rigid inclusions (respectively insulating or superconducting inclusions). Finally, note that although the user has a lot of freedom in terms of geometry (inclusion shapes, positions, orientations, interpenetration), special algorithms do need to be used to generate complex meshes, and these need to be systematically scrutinized to ensure overall good mesh quality, which is not always possible with very flat inclusions. One way to remedy this is to use interface elements to model the inclusions as perfectly flat (see perspectives). 
On the other hand, FFT grids are very easy to generate: a triple for-loop can be used to go through each voxel and assign an integer associated with the desired phase. Here, this was done using a simple home-made python script. However, this simplicity comes at a cost, namely a reduced freedom in the potentially usable inclusion orientations. Indeed, the Cartesian nature of the grid does generally restrict the possibilities to cylinders with axis parallel to one of the coordinate axes, because the inclusions are one voxel thick. Introducing an inclusion at $45^{\circ}$ with respect to one of the coordinate axes would result in a "staircase" that may not transmit fluxes (either stresses or heat fluxes) well. Note that it has been shown in Gasnier et al. [20] that certain FFT schemes are in fact able to correctly compute the stress field around a single disk-like inclusion with normal parallel to $\boldsymbol{e}_{1}+\boldsymbol{e}_{2}+\boldsymbol{e}_{3}$, but they also showed that the thickness of the cracks should not be lower than 1.5 voxels for randomly oriented cracks. As for running times, they are plotted in figures 12 and 13 for voids/insulating inclusions and rigid/superconducting inclusions respectively. Both graphs show a linear trend in log-log scale, but there are some fundamental differences.
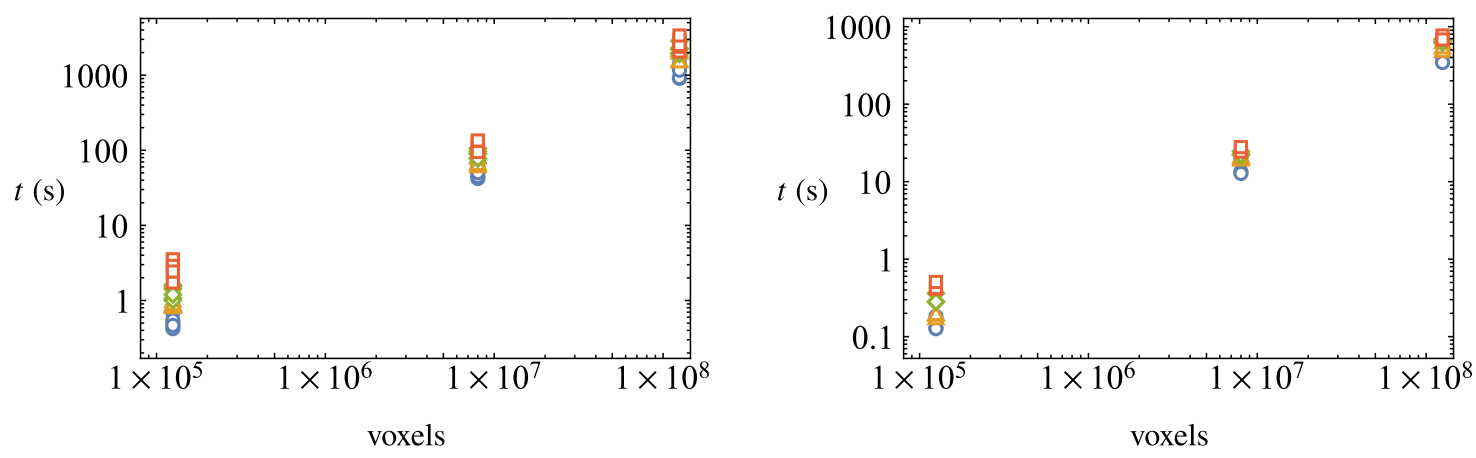

$$
\circ \epsilon=0.2 \Delta \epsilon=0.4 \quad \diamond \epsilon=0.6 \quad \square \epsilon=0.8
$$

Figure 12: FFT computation time as a function of grid size. Left: linear elastic composites with flat voids; right: conducting composites with flat insulating inclusions.

For voids/insulating inclusions (figure 12), the different simulations are well ordered according to the value of the DDP for each grid size, while for rigid/superconducting inclusions (figure 13) there does not seem to be any particular order. More striking is the fact that computation times for rigid/superconducting inclusions are systematically about an order of magnitude higher than for voids/insulating inclusions. Longer computation times were obtained because the algorithm was having trouble converging, even though the contrast was much lower for these simulations. These 

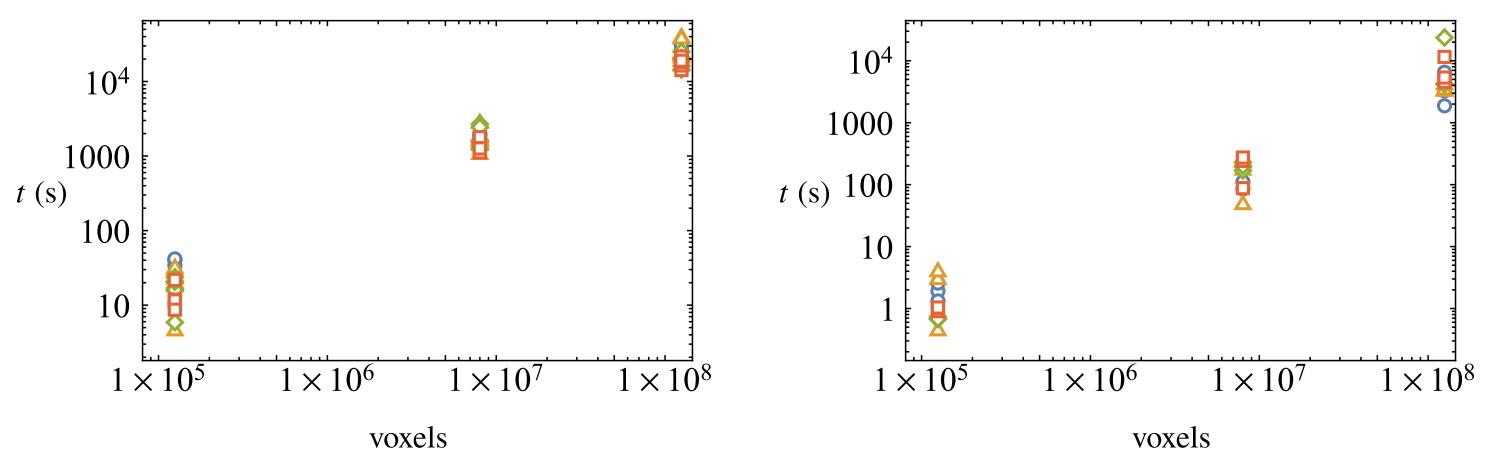

$\circ \epsilon=0.2 \Delta \epsilon=0.4 \quad \diamond \epsilon=0.6 \quad \square \epsilon=0.8$

Figure 13: FFT computation time as a function of grid size. Left: linear elastic composites with flat rigid inclusions; right: conducting composites with flat superconducting inclusions.

results, together with figures 10 and 11, suggest that the scheme implemented in AMITEX_FFTP is probably not well-suited for the computation of the in-plane properties of composites with disks of very high moduli (either elastic moduli or conductivities). That being said, it is worth noting that very low running times (often below $1 \mathrm{~s}$ ) were obtained for voids/insulating inclusions with the coarsest grids, and they still yielded some very good results (see the out-of-plane Young's modulus in figure 8). Additionally, even the $200^{3}$ grids had running times on the order of a couple of minutes for voids/insulating inclusions, and they yielded excellent results, which shows that in this case, this method does outperform FEM, which required at least one hour. Finally, note that there is not much freedom with respect to boundary conditions with this method, although some tricks are possible (for example using a one voxel thick layer of either a very soft or a very stiff material on the outside can approximate uniform boundary conditions).

\section{Conclusion}

In the present contribution, a somewhat original presentation has been laid down for the analytical estimation of the effective properties of composites with parallel flat inclusions. It has the advantage of being slightly more direct than previously published ones, and of underlining the similarities between the two dual kinds of contrasts, namely infinite and vanishing moduli for the inclusions with respect to the matrix. Numerical simulations have been performed to assess the predictions of five classical homogenization schemes for both linear elasticity and conduction. 
For the former, flat voids and flat rigid inclusions have been considered, while insulating and superconducting inclusions were used for the latter. In order to strengthen the conclusions, two very different numerical methods were used, namely the FEM with the code Cast $3 \mathrm{M}$ and the FFT-based method with the software AMITEX_FFTP.

The main conclusions are as follows. First, the good performance of the differential scheme has been confirmed in all cases. The only cases where the conclusion is not so cut and dry are those of rigid inclusions and superconducting inclusions, whereby FFT computations did not converge very well due to percolation phenomena. Thankfully, FEM computations did not suffer from this drawback, which allows for definite conclusions under the present hypotheses. Since the same scheme was identified in the case of linear elasticity and conduction, it will be possible to use it when considering coupled problems, such as the hydromechanical behavior of rocks. In terms of numerical methods, each one has its pros and cons: excellent results have been obtained in a matter of minutes with the FFT software for the out-of-plane components when the stiffness/conductivity of the inclusions is much smaller than that of the matrix, but the same method was clearly not very efficient for the in-plane components when contrast is reversed due to percolation. It would be interesting to investigate these phenomena using a different software to ascertain whether they are related to this specific implementation (AMITEX_FFTP), or to the method as a whole. The simplicity of the discretization procedure does make it an attractive method, but it also restricts the possible geometries. FEM is and remains a very robust tool, which was yet again confirmed by its lack of sensitivity to percolation phenomena. Several options are available to improve the geometrical description of the disks and possibly reduce the computation times, most notably XFEM and interface elements seem very attractive.

It should be recalled that the resulting choice of a scheme only holds here in the context of parallel disks, and other cases should be investigated independently. From the theoretical standpoint, random and axisymmetric distributions of disks have indeed already been investigated in the past, and the interested reader is referred to Fritsch et al. [19] for mechanical properties, and to Abdalrahman et al. [1] and Kiefer et al. [26] for transport properties. 


\section{Appendix A. Kernels of Hill's tensors for a disk in a linear elastic material}

Consider a spheroidal region of space with semi-minor axis in the direction of the unit vector n. Assuming the space to be filled with a linear elastic material with stiffness tensor $\mathbb{C}^{0}$, then Hill's tensor of the ellipsoid $\mathcal{E}$ has the following expression (see Barthelemy [3]):

$$
\mathbb{P}^{\mathcal{E}, 0}=\frac{\operatorname{det} \boldsymbol{A}}{4 \pi} \int_{\|\boldsymbol{\xi}\|=1} \frac{\Gamma_{4}(\boldsymbol{\xi})}{\|\boldsymbol{A} \cdot \boldsymbol{\xi}\|^{3}} \mathrm{~d} S_{\boldsymbol{\xi}}
$$

where $\boldsymbol{A}$ defines the ellipsoid through the equation $\left\|\boldsymbol{A}^{-1} \cdot \boldsymbol{\xi}\right\| \leq 1$, and $\Gamma_{4}$ is given by:

$$
\Gamma_{4}(\boldsymbol{\xi})=\stackrel{s}{\otimes}^{\otimes}\left(\boldsymbol{\xi} \cdot \mathbb{C}^{0} \cdot \boldsymbol{\xi}\right)^{-1} \stackrel{s}{\otimes} \boldsymbol{\xi}
$$

The subscript "4" refers to the fact that this tensor function has rank 4. As explained in Barthelemy [3], a change of variable allows one to show that:

$$
\lim _{X \rightarrow 0} \mathbb{P}^{\mathcal{E}, 0}=\Gamma_{4}(\boldsymbol{n})
$$

Thus, $\Gamma_{4}(\boldsymbol{n})$ is the $O(1)$ term in the expansion of $\mathbb{P}^{\mathcal{E}, 0}$ in powers of $X$. It is thus the Hill's tensor for a disk $(X \rightarrow 0)$ with the reference stiffness $\mathbb{C}^{0}$. Let $\boldsymbol{C}$ be defined by $\boldsymbol{C}=\left(\boldsymbol{n} \cdot \mathbb{C}^{0} \cdot \boldsymbol{n}\right)^{-1}$, and let $\boldsymbol{l}$ and $\boldsymbol{m}$ be two vectors such that $(\boldsymbol{l}, \boldsymbol{m}, \boldsymbol{n})$ is orthonormal. Then, by definition of the symmetric tensor product, one has:

$$
\left(\Gamma_{4}(\boldsymbol{n})\right)_{i j k l}=\frac{1}{4}\left(n_{i} C_{j k} n_{l}+n_{j} C_{i k} n_{l}+n_{i} C_{j l} n_{k}+n_{j} C_{i l} n_{k}\right)
$$

Then, for any two vectors $\boldsymbol{u}, \boldsymbol{v}$ orthogonal to $\boldsymbol{n}, \boldsymbol{u} \otimes \boldsymbol{v}$ is in the kernel of $\Gamma_{4}(\boldsymbol{n})$, since:

$$
\left(\Gamma_{4}(\boldsymbol{n}):(\boldsymbol{u} \otimes \boldsymbol{v})\right)_{i j}=\frac{1}{4}\left(n_{i} C_{j k} n_{l}+n_{j} C_{i k} n_{l}+n_{i} C_{j l} n_{k}+n_{j} C_{i l} n_{k}\right) u_{l} v_{k}
$$

and all the terms either have a $\boldsymbol{u} \cdot \boldsymbol{n}=0$ or a $\boldsymbol{v} \cdot \boldsymbol{n}=0$ factor. Of course, this is also true of $\boldsymbol{u} \stackrel{s}{\otimes} \boldsymbol{v}$. Thus, it appears that $\mathcal{V}_{i p}^{2}=\operatorname{span}(\boldsymbol{l} \otimes \boldsymbol{l}, \boldsymbol{m} \otimes \boldsymbol{m}, \boldsymbol{l} \stackrel{s}{\otimes} \boldsymbol{m}) \subset \operatorname{Ker} \Gamma_{4}(\boldsymbol{n})$. As a consequence, $\operatorname{dim} \operatorname{Ker} \Gamma_{4}(\boldsymbol{n}) \geq 3$. Next, we show that actually $\mathcal{V}_{i p}^{2}=\operatorname{Ker} \Gamma_{4}(\boldsymbol{n})$.

In order to do that, it is useful to consider the second Hill's tensor (Hill [24]):

$$
\mathbb{Q}^{\mathcal{E}, 0}=\mathbb{C}^{0}-\mathbb{C}^{0}: \mathbb{P}^{\mathcal{E}, 0}: \mathbb{C}^{0}
$$


Indeed, the $O(1)$ term of its expansion in powers of $X$ reads $\Lambda_{4}(\boldsymbol{n})=\mathbb{C}^{0}-\mathbb{C}^{0}: \Gamma_{4}(\boldsymbol{n}): \mathbb{C}^{0}$, but then:

$$
\mathbb{C}^{0}: \Gamma_{4}(\boldsymbol{n}): \mathbb{C}^{0}=\left(\mathbb{C}^{0} \cdot \boldsymbol{n}\right) \cdot\left(\boldsymbol{n} \cdot \mathbb{C}^{0} \cdot \boldsymbol{n}\right)^{-1} \cdot\left(\boldsymbol{n} \cdot \mathbb{C}^{0}\right)
$$

so that taking the dot product with $\boldsymbol{n}$ yields:

$$
\left(\mathbb{C}^{0}: \Gamma_{4}(\boldsymbol{n}): \mathbb{C}^{0}\right) \cdot \boldsymbol{n}=\mathbb{C}^{0} \cdot \boldsymbol{n}
$$

As a consequence, $\left(\mathbb{C}^{0}: \Gamma_{4}(\boldsymbol{n}): \mathbb{C}^{0}\right):(\boldsymbol{n} \otimes \boldsymbol{v})=\mathbb{C}^{0}:(\boldsymbol{n} \otimes \boldsymbol{v})$ for any vector $\boldsymbol{v}$. This also holds when replacing the tensor product with the symmetric tensor product because $\mathbb{C}^{0}: \Gamma_{4}(\boldsymbol{n}): \mathbb{C}^{0}$ has the minor symmetries. Thus, $\mathcal{V}_{\text {oop }}^{2}=\operatorname{span}(\boldsymbol{n} \otimes \boldsymbol{n}, \boldsymbol{n} \stackrel{s}{\otimes} \boldsymbol{l}, \boldsymbol{n} \stackrel{s}{\otimes} \boldsymbol{m}) \subset \operatorname{Ker} \Lambda_{4}(\boldsymbol{n})$, and thus $\operatorname{dim} \operatorname{Ker} \Lambda_{4}(\boldsymbol{n}) \geq 3$. Note that $\Lambda_{4}(\boldsymbol{n})$ is the second Hill's tensor for a disk with the reference stiffness $\mathbb{C}^{0}$.

To finish the proof, note that any symmetric second-rank tensor $\boldsymbol{\alpha}$ in $\operatorname{Ker} \Lambda_{4}(\boldsymbol{n})$ is an eigenvector of $\mathbb{C}^{0}: \Gamma_{4}(\boldsymbol{n})$ with eigenvalue 1 . Since $\mathbb{C}^{0}$ is invertible, it follows that $\operatorname{Rank} \Gamma_{4}(\boldsymbol{n}) \geq 3$. According to the rank-nullity theorem, $\operatorname{Rank} \Gamma_{4}(\boldsymbol{n})+\operatorname{dim} \operatorname{Ker} \Gamma_{4}(\boldsymbol{n})=6$, but both terms are greater than or equal to 3, so one necessarily has $\operatorname{Rank} \Gamma_{4}(\boldsymbol{n})=\operatorname{dim} \operatorname{Ker} \Gamma_{4}(\boldsymbol{n})=3$. Finally, $\mathcal{V}_{i p}^{2} \subset \operatorname{Ker} \Gamma_{4}(\boldsymbol{n})$ and both linear subsets have the same dimension, so that $\mathcal{V}_{i p}^{2}=\operatorname{Ker} \Gamma_{4}(\boldsymbol{n})$.

Similarly, any symmetric second-rank tensor $\boldsymbol{\alpha}$ in $\operatorname{Ker} \Gamma_{4}(\boldsymbol{n})$ is an eigenvector of $\mathbb{S}^{0}: \Lambda_{4}(\boldsymbol{n})$ with eigenvalue 1. Consequently, Rank $\Lambda_{4}(\boldsymbol{n}) \geq 3$, and application of the rank-nullity theorem again shows that $\operatorname{Rank} \Lambda_{4}(\boldsymbol{n})=\operatorname{dim} \operatorname{Ker} \Lambda_{4}(\boldsymbol{n})=3$. Thus, $\mathcal{V}_{\text {oop }}^{2} \subset \operatorname{Ker} \Lambda_{4}(\boldsymbol{n})$, and both linear subsets have the same dimension, so that $\mathcal{V}_{\text {oop }}^{2}=\operatorname{Ker} \Lambda_{4}(\boldsymbol{n})$.

Thus, the $O(1)$ terms in the expansions of $\mathbb{P}^{\mathcal{E}, 0}$ and $\mathbb{Q}^{\mathcal{E}, 0}$ are both singular, their kernels have dimension 3, and the linear space of symmetric second-rank tensors is their direct sum.

\section{Appendix B. Concentration tensor for a flat rigid inclusion in a transversely isotropic matrix}

Using identical conventions to Bluthe et al. [7], an orthonormal basis $\mathscr{B}=\left(\boldsymbol{e}_{1}, \boldsymbol{e}_{2}, \boldsymbol{e}_{3}\right)$ of the Euclidean space is introduced, and the Mandel representation of second-rank and fourth-rank tensors is adopted. The reference material is assumed to be transversely isotropic with stiffness tensor $\mathbb{C}^{0}$, and its axis of transverse isotropy is taken to be parallel to $\boldsymbol{e}_{3}$, so that its Mandel 
representation may be expressed as:

$$
\left[\mathbb{C}^{0}\right]_{\mathscr{B}}=\left(\begin{array}{cccccc}
M_{1} & M_{1}-2 \mu_{12} & \lambda_{13} & 0 & 0 & 0 \\
M_{1}-2 \mu_{12} & M_{1} & \lambda_{13} & 0 & 0 & 0 \\
\lambda_{13} & \lambda_{13} & M_{3} & 0 & 0 & 0 \\
0 & 0 & 0 & 2 \mu_{13} & 0 & 0 \\
0 & 0 & 0 & 0 & 2 \mu_{13} & 0 \\
0 & 0 & 0 & 0 & 0 & 2 \mu_{12}
\end{array}\right)
$$

$M_{1}$ and $M_{3}$ will be referred to as the in-plane and out-of-plane P-wave moduli respectively, $\mu_{12}$ and $\mu_{13}$ as the in-plane and out-of-plane shear moduli respectively, and $\lambda_{13}$ as the out-of-plane Lamé's first parameter.

Derivation of the concentration tensor requires the expansion to first order in $X$ of Hill's tensor:

$$
\mathbb{P}^{\mathcal{E}, 0}=\Gamma_{4}(\boldsymbol{n})+X \Pi+o(X)
$$

As shown in Appendix $\mathrm{A}, \Gamma_{4}(\boldsymbol{n})$ is singular with kernel $\mathcal{V}_{i p}^{2}$, so that $\lim _{X \rightarrow 0}\left(\mathbb{P}^{\mathcal{E}, 0}\right)^{-1}$ is computed by inverting the corresponding square submatrix of $\Pi$ (see Barthelemy [3]). The tensor $\Pi$ can be obtained from Laws [28]. For a spheroid with axis of revolution parallel to $\boldsymbol{n}$, Hill's tensor is also transversely isotropic, and its components can be obtained from two integrals given in Laws [28]. The author also gives their expansion to first order in $X$, which allows to easily compute the required components of $\Pi$ :

$$
\left\{\begin{array}{l}
\Pi_{1111}=\frac{\pi}{16}\left(\frac{3}{\mu_{13} \gamma}+\frac{3}{\sqrt{M_{1} M_{3} \gamma}}+\frac{1}{\sqrt{\mu_{12} \mu_{13}}}\right) \\
\Pi_{1122}=\frac{\pi}{16}\left(\frac{1}{\mu_{13} \gamma}+\frac{1}{\sqrt{M_{1} M_{3} \gamma}}-\frac{1}{\sqrt{\mu_{12} \mu_{13}}}\right)
\end{array}\right.
$$

with:

$$
\gamma=\sqrt{\frac{M_{1}}{\mu_{13}}+2 \sqrt{\frac{M_{1}}{M_{3}}}-\frac{\lambda_{13}\left(\lambda_{13}+2 \mu_{13}\right)}{M_{3} \mu_{13}}}
$$


The Mandel representation of $\mathbb{V}^{d, 0}$ then reads:

$$
\left[\mathbb{V}^{d, 0}\right]_{\mathscr{B}}=\left(\begin{array}{cccccc}
\frac{A+B}{2} & \frac{A-B}{2} & 0 & 0 & 0 & 0 \\
\frac{A-B}{2} & \frac{A+B}{2} & 0 & 0 & 0 & 0 \\
0 & 0 & 0 & 0 & 0 & 0 \\
0 & 0 & 0 & 0 & 0 & 0 \\
0 & 0 & 0 & 0 & 0 & 0 \\
0 & 0 & 0 & 0 & 0 & B
\end{array}\right)
$$

with:

$$
\left\{\begin{array}{l}
A=\frac{1}{\Pi_{1111}+\Pi_{1122}} \\
B=\frac{1}{\Pi_{1111}-\Pi_{1122}}
\end{array}\right.
$$

As can be seen from (B.5) and the effective stiffness tensors (24), (29) and (30), $\lambda_{13}, M_{3}$ and $\mu_{13}$ are not affected by $\mathbb{V}^{d, 0}$. For all the schemes, the reference stiffness must reduce to the stiffness of the matrix for $\epsilon=0$, so $\lambda_{13}, M_{3}$ and $\mu_{13}$ are actually equal to their values for $\epsilon=0$, i.e. $\lambda_{13}=M_{m}-2 \mu_{m}, M_{3}=M_{m}$ and $\mu_{13}=\mu_{m}$. Then, (B.3) reduces to:

$$
\left\{\begin{array}{l}
\Pi_{1111}=\frac{\pi}{16}\left(\frac{3}{\mu_{m} \gamma}+\frac{3}{\sqrt{M_{1} M_{m}} \gamma}+\frac{1}{\sqrt{\mu_{12} \mu_{m}}}\right) \\
\Pi_{1122}=\frac{\pi}{16}\left(\frac{1}{\mu_{m} \gamma}+\frac{1}{\sqrt{M_{1} M_{m}} \gamma}-\frac{1}{\sqrt{\mu_{12} \mu_{m}}}\right)
\end{array}\right.
$$

with:

$$
\gamma=\sqrt{2\left(1+\sqrt{\frac{M_{1}}{M_{m}}}\right)+\frac{M_{m}}{\mu_{m}}\left(\frac{M_{1}}{M_{m}}-1\right)}
$$

These are the equations needed for the self-consistent and differential approximations. For the dilute and Mori-Tanaka approximations and for the PCW bound, the reference material actually has the stiffness $\mathbb{C}^{m}$, so that $M_{1}=M_{m}$ and $\mu_{12}=\mu_{m}$, in which case $\gamma=2$ and:

$$
\left\{\begin{array}{l}
\Pi_{1111}=\frac{\pi}{32}\left(\frac{3}{M_{m}}+\frac{5}{\mu_{m}}\right) \\
\Pi_{1122}=\frac{\pi}{32}\left(\frac{1}{M_{m}}-\frac{1}{\mu_{m}}\right)
\end{array}\right.
$$




\section{Appendix C. Concentration tensor for an insulating/superconducting disk in a transversely isotropic matrix}

The developments are similar to those of Appendix A and Appendix B, but much simplified by the lower ranks of tensors in the case of linear conduction. Denoting $\boldsymbol{K}^{0}$ the reference conductivity, the Hill's tensor now reads:

$$
\boldsymbol{P}^{\mathcal{E}, 0}=\frac{\operatorname{det} \boldsymbol{A}}{4 \pi} \int_{\|\boldsymbol{\xi}\|=1} \frac{\Gamma_{2}(\boldsymbol{\xi})}{\|\boldsymbol{A} \cdot \boldsymbol{\xi}\|^{3}} \mathrm{~d} S_{\boldsymbol{\xi}} ; \quad \Gamma_{2}(\boldsymbol{\xi})=\frac{\boldsymbol{\xi} \otimes \boldsymbol{\xi}}{\boldsymbol{\xi} \cdot \boldsymbol{K}^{0} \cdot \boldsymbol{\xi}}
$$

where the subscript "2" has been used since $\Gamma_{2}(\xi)$ is a second rank tensor function. In analogy with linear elasticity, its expansion to first order in $X$ then reads:

$$
\boldsymbol{P}^{\mathcal{E}, 0}=\Gamma_{2}(\boldsymbol{n})+X \varpi+o(X)
$$

It is clear that $\operatorname{Ker} \Gamma_{2}(\boldsymbol{n})$ is exactly $\mathcal{V}_{i p}^{1}=\operatorname{span}(\boldsymbol{l}, \boldsymbol{m})$. The second Hill's tensor reads:

$$
\boldsymbol{Q}^{\mathcal{E}, 0}=\boldsymbol{K}^{0}-\boldsymbol{K}^{0} \cdot \boldsymbol{P}^{\mathcal{E}, 0} \cdot \boldsymbol{K}^{0}
$$

so that the $O(1)$ term in its expansion in powers of $X$ reads:

$$
\Lambda_{2}(\boldsymbol{n})=\boldsymbol{K}^{0}-\frac{\left(\boldsymbol{K}^{0} \cdot \boldsymbol{n}\right) \otimes\left(\boldsymbol{n} \cdot \boldsymbol{K}^{0}\right)}{\boldsymbol{n} \cdot \boldsymbol{K}^{0} \cdot \boldsymbol{n}}
$$

and taking the dot product with $\boldsymbol{n}$ yields $\Lambda_{2}(\boldsymbol{n}) \cdot \boldsymbol{n}=0$. In analogy with linear elasticity, any vector of $\operatorname{Ker} \Gamma_{2}(\boldsymbol{n})$ is an eigenvector with eigenvalue one of $\boldsymbol{R}^{0} \cdot \Lambda_{2}(\boldsymbol{n})$, so that $\operatorname{Rank} \Lambda_{2}(\boldsymbol{n}) \geq 2$, and $\operatorname{Rank} \Lambda_{2}(\boldsymbol{n})=2$ by application of the rank-nullity theorem. As a consequence, $\operatorname{dim} \operatorname{Ker} \Lambda_{2}(\boldsymbol{n})=1$, but since $\mathcal{V}_{\text {oop }}^{1}=\operatorname{span}(\boldsymbol{n}) \subset \operatorname{Ker} \Lambda_{2}(\boldsymbol{n})$, one actually has $\mathcal{V}_{\text {oop }}^{1}=\operatorname{Ker} \Lambda_{2}(\boldsymbol{n})$.

A general expression for $\boldsymbol{P}^{\mathcal{E}, 0}$ may be obtained for a spheroid whose axis of revolution is parallel to the direction of transverse isotropy of the reference material (along $\boldsymbol{e}_{3}$ in the following). Denoting $K_{0}=K_{11}^{0}=K_{22}^{0}$ and $V=\frac{K_{33}^{0}}{K_{0}}$, the two independent components of the Hill's tensor are given by:

$$
P_{11}^{\mathcal{S}, 0}=P_{22}^{\mathcal{S}, 0}=\frac{1}{2 K_{0}}\left(1-F\left(\frac{X}{\sqrt{V}}\right)\right) ; \quad P_{33}^{\mathcal{S}, 0}=\frac{1}{K_{0} V} F\left(\frac{X}{\sqrt{V}}\right)
$$


with $F(x)=\frac{1}{1-x^{2}}-\frac{x}{\left(1-x^{2}\right)^{3 / 2}} \arccos x$ (see Parnell [32] for instance). Here the superscript $\mathcal{S}$ was used to stress the fact that the inclusion is a spheroid. The tensor $\varpi$ is then readily obtained:

$$
[\varpi]_{\mathscr{B}}=\frac{1}{K_{0}}\left(\begin{array}{ccc}
\frac{\pi}{4 \sqrt{V}} & 0 & 0 \\
0 & \frac{\pi}{4 \sqrt{V}} & 0 \\
0 & 0 & -\frac{\pi}{2 V^{3 / 2}}
\end{array}\right)
$$

and $\boldsymbol{U}^{d, 0}$ follows:

$$
\left[\boldsymbol{U}^{d, 0}\right]_{\mathscr{B}}=\frac{4 \sqrt{V}}{\pi} K_{0}\left(\begin{array}{ccc}
1 & 0 & 0 \\
0 & 1 & 0 \\
0 & 0 & 0
\end{array}\right)
$$

Finally, the $O(X)$ term in the expansion of $Q^{\mathcal{S}, 0}$ reads:

$$
\left[-\boldsymbol{K}^{0} \cdot \varpi \cdot \boldsymbol{K}^{0}\right]_{\mathscr{B}}=-K_{0}\left(\begin{array}{ccc}
\frac{\pi}{4 \sqrt{V}} & 0 & 0 \\
0 & \frac{\pi}{4 \sqrt{V}} & 0 \\
0 & 0 & -\frac{\pi}{2} \sqrt{V}
\end{array}\right)
$$

and $\boldsymbol{V}^{d, 0}$ follows:

$$
\left[\boldsymbol{V}^{d, 0}\right]_{\mathscr{B}}=\frac{2}{\pi \sqrt{V}} \frac{1}{K_{0}}\left(\begin{array}{ccc}
0 & 0 & 0 \\
0 & 0 & 0 \\
0 & 0 & 1
\end{array}\right)
$$

These expressions are used for the self-consistent and differential schemes, while $V$ is set to 1 for the dilute and Mori-Tanaka schemes, as well as for the PCW bound.

\section{Appendix D. Effective moduli and conductivities}

All the analytical estimates are summarized in tables D.2 and D.3 for completeness. In D.2, the tensors $\mathbb{U}^{d, P C W}$ and $\mathbb{V}^{d, P C W}$ are computed from $\mathbb{Q}^{P C W}=\frac{1}{1-f}\left(\mathbb{Q}^{I, m}-f \mathbb{Q}^{\text {sphere,m }}\right)$ and $\mathbb{P}^{P C W}=\frac{1}{1-f}\left(\mathbb{P}^{I, m}-f \mathbb{P}^{\text {sphere, } m}\right)$ by taking the limits (12) and (21) respectively. The conductivities are given explicitly since the calculations may be carried out to the end, but only the tensorial results 
are given for elasticity for the sake of brevity. It is clear from table D.3 that all the conductivity estimates yield the dilute scheme upon expansion to first order in $\epsilon$, as they should.

\begin{tabular}{c|c|c}
\multicolumn{2}{c}{ Table D.2: Analytical effective compliance/stiffness tensors. } \\
Scheme & Flat voids & Flat rigid inclusions \\
\hline Dilute scheme & $\mathbb{S}^{d i l}=\mathbb{S}^{m}+\frac{4 \pi}{3} \epsilon \mathbb{U}^{d, m}$ & $\mathbb{C}^{d i l}=\mathbb{C}^{m}+\frac{4 \pi}{3} \epsilon \mathbb{V}^{d, m}$ \\
Mori-Tanaka scheme & $\mathbb{S}^{M T}=\mathbb{S}^{m}+\frac{4 \pi}{3} \epsilon \mathbb{U}^{d, m}$ & $\mathbb{C}^{M T}=\mathbb{C}^{m}+\frac{4 \pi}{3} \epsilon \mathbb{V}^{d, m}$ \\
Self-consistent scheme & $\mathbb{S}^{s c}=\mathbb{S}^{m}+\frac{4 \pi}{3} \epsilon \mathbb{U}^{d, s c}$ & $\mathbb{C}^{s c}=\mathbb{C}^{m}+\frac{4 \pi}{3} \epsilon \mathbb{V}^{d, s c}$ \\
Differential scheme & $\frac{\mathrm{d}}{\mathrm{de}} \mathbb{S}^{d i f}=\frac{4 \pi}{3} \mathbb{U}^{d, d i f}$ & $\frac{\mathrm{d}}{\mathrm{de}} \mathbb{C}^{d i f}=\frac{4 \pi}{3} \mathbb{V}^{d, d i f}$ \\
PCW bound & $\mathbb{S}^{P C W}=\mathbb{S}^{m}+\frac{4 \pi}{3} \epsilon \mathbb{U}^{d, P C W}$ & $\mathbb{C}^{P C W}=\mathbb{C}^{m}+\frac{4 \pi}{3} \epsilon \mathbb{V}^{d, P C W}$
\end{tabular}

Tensorial expressions were preferred here because they are more compact, and because they do possess all the information needed to infer the elastic moduli. Note that explicit expressions exist for these moduli in all cases, except that of the self-consistent scheme. For the dilute and Mori-Tanaka schemes, the expressions are easily derived, for the PCW bound they may be found in Ponte Castaneda and Willis [33] although they require more calculation, and for the differential scheme the differential equations satisfied by the elastic moduli in the case of flat voids have been given by Salganik [34] and their closed-form solution can be found in Zimmerman [40]. The implicit equation of the self-consistent scheme and the differential equation of the differential scheme in the case of rigid inclusions were solved numerically in the present paper. 
Table D.3: Analytical effective conductivities.

\begin{tabular}{c|c|c} 
Scheme & Flat insulating inclusions & Flat superconducting inclusions \\
\hline Dilute scheme & $\frac{K_{33}}{K_{m}}=1-\frac{8}{3} \epsilon$ & $\frac{K_{11}}{K_{m}}=\frac{K_{22}}{K_{m}}=1+\frac{16}{3} \epsilon$ \\
Mori-Tanaka scheme & $\frac{K_{33}}{K_{m}}=\left(1+\frac{8}{3} \epsilon\right)^{-1}$ & $\frac{K_{11}}{K_{m}}=\frac{K_{22}}{K_{m}}=1+\frac{16}{3} \epsilon$ \\
Self-consistent scheme & $\frac{K_{33}}{K_{m}}=\left(\sqrt{1+\frac{16}{9} \epsilon^{2}}-\frac{4}{3} \epsilon\right)^{2}$ & $\frac{K_{11}}{K_{m}}=\frac{K_{22}}{K_{m}}=\left(\sqrt{1+\frac{64}{9} \epsilon^{2}}-\frac{8}{3} \epsilon\right)^{-2}$ \\
Differential scheme & $\frac{K_{33}}{K_{m}}=\left(1+\frac{4}{3} \epsilon\right)^{-2}$ & $\frac{K_{11}}{K_{m}}=\frac{K_{22}}{K_{m}}=\left(1+\frac{8}{3} \epsilon\right)^{2}$ \\
PCW bound & $\frac{K_{33}}{K_{m}}=\left(1-\frac{16}{9} \epsilon\right) /\left(1+\frac{8}{9} \epsilon\right)$ & $\frac{K_{11}}{K_{m}}=\frac{K_{22}}{K_{m}}=\left(1+\frac{32}{9} \epsilon\right) /\left(1-\frac{16}{9} \epsilon\right)$
\end{tabular}

\section{Acknowledgment}

The authors gratefully acknowledge financial support from CEA and EDF/ERMES.

\section{References}

[1] T. Abdalrahman, S. Scheiner, and C. Hellmich. Is trabecular bone permeability governed by molecular orderinginduced fluid viscosity gain? Arguments from re-evaluation of experimental data in the framework of homogenization theory. Journal of Theoretical Biology, 365:433-444, jan 2015. doi: 10.1016/j.jtbi.2014.10.011.

[2] J.-F. Barthelemy. Approche micromécanique de la rupture et de la fissuration dans les géomatériaux. PhD thesis, Ecole des Ponts ParisTech, 2005. URL https://pastel . archives-ouvertes.fr/pastel-00001296/.

[3] J.-F. Barthelemy. Compliance and Hill polarization tensor of a crack in an anisotropic matrix. International Journal of Solids and Structures, 46(22):40644072, November 2009. ISSN 0020-7683. doi: 10.1016/j.ijsolstr.2009.08.003. URL http://www. sciencedirect.com/science/article/pii/S0020768309002893.

[4] J.-F. Barthelemy. Effective permeability of media with a dense network of long and micro fractures. Transport in porous media, 76(1):153-178, 2009. URL http://www. springerlink. com/index/92x601894jv33520.pdf.

[5] P. Blümling, F. Bernier, P. Lebon, and C. D. Martin. The excavation damaged zone in clay formations time-dependent behaviour and influence on performance assessment. Physics and Chemistry of the Earth, Parts A/B/C, 32(8):588-599, 2007. URL http://www.sciencedirect.com/science/article/pii/S1474706506002075. 
[6] J. Bluthe, B. Bary, and E. Lemarchand. Micromechanical modeling of the Compression of the Damaged Zone experiment in the Callovo-Oxfordian formation. Advances in Geosciences, 45:25-33, jul 2018. doi: 10.5194/adgeo-45-25-2018.

[7] J. Bluthe, B. Bary, and E. Lemarchand. Closure of parallel cracks: Micromechanical estimates versus finite element computations. European Journal of Mechanics - A/Solids, 81:103952, may 2020. doi: 10.1016/j.euromechsol.2020.103952.

[8] H. Bock, B. Dehandschutter, C. D. Martin, M. Mazurek, A. de Haller, F. Skoczylas, and C. Davy. Self-sealing of fractures in argillaceous formations in the context of geological disposal of radioactive waste, Review and Synthesis. Technical report, Nuclear Energy Agency, 2010.

[9] B. Budiansky and R. J. O’Connell. Elastic moduli of a cracked solid. International journal of Solids and structures, 12(2):81-97, 1976.

[10] L. Charpin and A. Ehrlacher. Estimating the poroelastic properties of cracked materials. Acta Mechanica, 225(9):2501-2519, September 2014. ISSN 1619-6937. doi: 10.1007/s00707-013-1082-0. URL https://doi.org/10.1007/s00707-013-1082-0.

[11] T. Dahm and T. Becker. On the elastic and viscous properties of media containing strongly interacting in-plane cracks. Pure and Applied Geophysics, 151(1):1-16, jan 1998. doi: 10.1007/s000240050102.

[12] C. A. Davy, F. Skoczylas, J.-D. Barnichon, and P. Lebon. Permeability of macro-cracked argillite under confinement: gas and water testing. Physics and Chemistry of the Earth, Parts A/B/C, 32(8):667-680, 2007. URL http://wWW. sciencedirect.com/science/article/pii/S1474706506002531.

[13] V. Deude, L. Dormieux, D. Kondo, and S. Maghous. Micromechanical approach to nonlinear poroelasticity: application to cracked rocks. Journal of engineering mechanics, 128(8):848-855, 2002. URL http: //ascelibrary.org/doi/abs/10.1061/(ASCE)0733-9399(2002) $128: 8$ (848).

[14] L. Dormieux and D. Kondo. Approche micromécanique du couplage perméabilité-endommagement. Comptes Rendus Mécanique, 332(2):135-140, 2004.

[15] L. Dormieux and D. Kondo. Micromechanics of Fracture and Damage. John Wiley \& Sons, 2016.

[16] L. Dormieux, D. Kondo, and F.-J. Ulm. Microporomechanics. John Wiley \& Sons, 2006.

[17] W. J. Drugan and J. R. Willis. A micromechanics-based nonlocal constitutive equation and estimates of representative volume element size for elastic composites. Journal of the Mechanics and Physics of Solids, 44(4): 497-524, apr 1996. doi: 10.1016/0022-5096(96)00007-5.

[18] J. D. Eshelby. The Determination of the Elastic Field of an Ellipsoidal Inclusion, and Related Problems. Proceedings of the Royal Society of London A: Mathematical, Physical and Engineering Sciences, 241(1226):376-396, August 1957. ISSN 1364-5021, 1471-2946. doi: 10.1098/rspa.1957.0133. URL http://rspa.royalsocietypublishing.org/content/241/1226/376.

[19] A. Fritsch, L. Dormieux, and C. Hellmich. Porous polycrystals built up by uniformly and axisymmetrically 
oriented needles: homogenization of elastic properties. Comptes Rendus Mécanique, 334(3):151-157, mar 2006. doi: 10.1016/j.crme.2006.01.008.

[20] J.-B. Gasnier, F. Willot, H. Trumel, D. Jeulin, and J. Besson. Thermoelastic properties of microcracked polycrystals. Part I: Adequacy of Fourier-based methods for cracked elastic bodies. International Journal of Solids and Structures, 155:248-256, dec 2018. doi: 10.1016/j.ijsolstr.2018.07.024.

[21] M. Ghayaza, F. Skoczylas, J. C. Robinet, and J. Talandier. Self-sealing capacity of macro-cracked argillite under confinement. In Poromechanics V: Proceedings of the Fifth Biot Conference on Poromechanics, pages 1580-1589, 2013. URL http://ascelibrary.org/doi/abs/10 .1061/9780784412992 . 187.

[22] V. Grechka. Comparison of the non-interaction and differential schemes in predicting the effective elastic properties of fractured media. International Journal of Fracture, 144(3):181-188, jul 2007. doi: 10.1007/s10704007-9088-z.

[23] Z. Hashin. The differential scheme and its application to cracked materials. Journal of the Mechanics and Physics of Solids, 36(6):719-734, January 1988. ISSN 0022-5096. doi: 10.1016/0022-5096(88)90005-1. URL http://www.sciencedirect.com/science/article/pii/0022509688900051.

[24] R. Hill. A self-consistent mechanics of composite materials. Journal of the Mechanics and Physics of Solids, 13(4):213-222, August 1965. ISSN 0022-5096. doi: 10.1016/0022-5096(65)90010-4. URL http://www. sciencedirect.com/science/article/pii/0022509665900104.

[25] S. J. Hollister and N. Kikuchi. A comparison of homogenization and standard mechanics analyses for periodic porous composites. Computational Mechanics, 10(2):73-95, 1992. doi: 10.1007/bf00369853.

[26] T. Kiefer, J. Füssl, H. Kariem, J. Konnerth, W. Gaggl, and C. Hellmich. A multi-scale material model for the estimation of the transversely isotropic thermal conductivity tensor of fired clay bricks. Journal of the European Ceramic Society, 40(15):6200-6217, dec 2020. doi: 10.1016/j.jeurceramsoc.2020.05.018.

[27] V. Kushch and A. Sangani. Conductivity of a composite containing uniformly oriented penny-shaped cracks or perfectly conducting discs. Proceedings of the Royal Society of London. Series A: Mathematical, Physical and Engineering Sciences, 456(1995):683-699, 2000.

[28] N. Laws. A note on penny-shaped cracks in transversely isotropic materials. Mechanics of Materials, 4(2): 209-212, jul 1985. doi: 10.1016/0167-6636(85)90017-1.

[29] E. Lemarchand, C. A. Davy, L. Dormieux, W. Chen, and F. Skoczylas. Micromechanics contribution to coupled transport and mechanical properties of fractured geomaterials. Transport in Porous Media, 79(3):335-358, jan 2009. doi: 10.1007/s11242-008-9326-5.

[30] S. Levasseur, F. Collin, R. Charlier, and D. Kondo. A micro-macro approach of permeability evolution in rocks excavation damaged zones. Computers and Geotechnics, 49:245-252, 2013.

[31] H. Moulinec and P. Suquet. A FFT-based numerical method for computing the mechanical properties of composites from images of their microstructures. In IUTAM Symposium on Microstructure-Property Interactions 
in Composite Materials, pages 235-246. Springer Netherlands, 1995.

[32] W. J. Parnell. The Hill and Eshelby tensors for ellipsoidal inhomogeneities in the Newtonian potential problem and linear elastostatics, 2015.

[33] P. Ponte Castaneda and J. R. Willis. The effect of spatial distribution on the effective behavior of composite materials and cracked media. Journal of the Mechanics and Physics of Solids, 43(12):1919-1951, dec 1995. doi: 10.1016/0022-5096(95)00058-q.

[34] R. L. Salganik. Mechanics of bodies with many cracks. Mechanics of Solids, 8(4):135-143, 1973.

[35] K. Vasylevskyi, B. Drach, and I. Tsukrov. On micromechanical modeling of orthotropic solids with parallel cracks. International Journal of Solids and Structures, 144145:46-58, July 2018. ISSN 0020-7683. doi: 10.1016/j.ijsolstr.2018.02.038. URL http://www.sciencedirect.com/science/article/pii/S0020768318300544.

[36] F. Willot, H. Trumel, and D. Jeulin. The thermoelastic response of cracked polycrystals with hexagonal symmetry. Philosophical Magazine, 99(5):606-630, nov 2018. doi: 10.1080/14786435.2018.1547432.

[37] F. Willot, R. Brenner, and H. Trumel. Elastostatic field distributions in polycrystals and cracked media. Philosophical Magazine, 100(6):661-687, dec 2019. doi: 10.1080/14786435.2019.1699669.

[38] C. L. Zhang. Experimental evidence for self-sealing of fractures in claystone. Physics and Chemistry of the Earth, Parts A/B/C, 36(17):1972-1980, 2011. URL http://www.sciencedirect.com/science/article/pii/S147470651100146X.

[39] C. L. Zhang. Sealing of fractures in claystone. Journal of Rock Mechanics and Geotechnical Engineering, 5(3): 214-220, 2013. URL http://wwW. sciencedirect.com/science/article/pii/S1674775513000334.

[40] R. W. Zimmerman. The effect of microcracks on the elastic moduli of brittle materials. Journal of materials science letters, 4(12):1457-1460, 1985. 